\title{
Inferring black hole spins and probing accretion/ejection flows in AGNs with the Athena X-ray Integral Field Unit
}

\author{
Didier Barret $^{1}$ and Massimo Cappi ${ }^{2}$ \\ 1 Université de Toulouse, CNRS, Institut de Recherche en Astrophysique et Planétologie, 9 Avenue du colonel Roche, \\ BP 44346, 31028 Toulouse Cedex 4, France \\ e-mail: dbarret@irap.omp.eu \\ 2 INAF-Osservatorio di Astrofisica e Scienza dello Spazio di Bologna (OAS), Via Piero Gobetti 93/3, 40129 Bologna, Italy
}

Received 1 May 2019 / Accepted 3 June 2019

\begin{abstract}
Context. Active galactic nuclei (AGNs) display complex X-ray spectra that exhibit a variety of emission and absorption features. These are commonly interpreted as a combination of (i) a relativistically smeared reflection component, resulting from the irradiation of an accretion disk by a compact hard X-ray source; (ii) one or several warm or ionized absorption components produced by AGN-driven outflows crossing our line of sight; and (iii) a nonrelativistic reflection component produced by more distant material. Disentangling these components via detailed model fitting could be used to constrain the black hole spin, geometry, and characteristics of the accretion flow, as well as of the outflows and surroundings of the black hole.

Aims. We investigate how a high-throughput high-resolution X-ray spectrometer such as the Athena X-ray Integral Field Unit (X-IFU) can be used to this aim, using the state-of-the-art reflection model relxill in a lamp-post geometrical configuration.

Methods. We simulated a representative sample of AGN spectra, including all necessary model complexities, as well as a range of model parameters going from standard to more extreme values, and considered X-ray fluxes that are representative of known AGN and quasar populations. We also present a method to estimate the systematic errors related to the uncertainties in the calibration of the X-IFU.

Results. In a conservative setting, in which the reflection component is computed self consistently by the relxill model from the preset geometry and no iron overabundance, the mean errors on the spin and height of the irradiating source are $<0.05$ and $\sim 0.2 R_{\mathrm{g}}$ (in units of gravitational radius). Similarly, the absorber parameters (column density, ionization parameter, covering factor, and velocity) are measured to an accuracy typically less than $\sim 5 \%$ over their allowed range of variations. Extending the simulations to include blueshifted ultra-fast outflows, we show that X-IFU could measure their velocity with statistical errors $<1 \%$, even for high-redshift objects (e.g., at redshifts $\sim 2.5$ ).

Conclusion. The simulations presented here demonstrate the potential of the X-IFU to understand how black holes are powered and how they shape their host galaxies. The accuracy in recovering the physical model parameters encoded in their X-ray emission is reached thanks to the unique capability of X-IFU to separate and constrain narrow and broad emission and absorption components.
\end{abstract}

Key words. black hole physics - accretion, accretion disks - radiation mechanisms: general - instrumentation: detectors quasars: supermassive black holes - X-rays: general

\section{Introduction}

$\mathrm{X}$-rays provide a unique opportunity to directly probe the innermost regions around AGN central black holes and are of utmost importance to modern studies of these objects. In fact, AGN spectral properties exhibiting numerous emission and absorption lines in X-rays, combined with the observed fast variability of both continuum and lines, provide unique tools to measure the velocities, the ionization states, the time variations and the geometries of the accretion or ejection flows surrounding supermassive black holes (SMBHs; Done 2010; Fabian 2016; Kaastra 2017a; Reynolds 2019).

With the advent of high-throughput and high-spectralresolution imaging and grating spectrometers on-board XMM-Newton and Chandra, the field has seen the flourishing of a number of detailed spectral and timing studies addressing two broad topics: (i) the SMBH-accretion disk systems via the relativistic reflection component from the accretion disk and the inferred SMBH spins (e.g., Tanaka et al. 1995; Nandra et al. 1997; Brenneman \& Reynolds 2006; Fabian et al. 2009; Zoghbi et al. 2010, 2019; De Marco et al. 2013; Brenneman
2013; Fabian et al. 2017; Kara et al. 2016; García et al. 2019a), and (ii) the study of AGN-driven outflows (from low-velocity warm absorbers to more extreme ultra-fast outflows; UFOs) thought to originate from the accretion disk, the inner broad line region, and/or the inner torus via a yet-unknown physical process (Reeves et al. 2003; Pounds et al. 2003; Blustin et al. 2005; Tombesi et al. 2010; Kaastra et al. 2014; Mehdipour et al. 2015; Nardini et al. 2015; Cappi et al. 2016; Parker et al. 2018a).

To progress on both these topics is of extreme importance with wide-ranging implications. For example, the spin of a black hole encodes information about its growth history, may play an active role in setting relativistic jets and energetic outflows shaping the evolution of its host galaxy, determines the radiative accretion efficiency, and sets the magnitude of some of the most extreme general relativistic phenomena observable in the Universe, such as gravitational redshift and light bending (Reynolds 2019). Similarly, massive outflows at very high velocity (up to $\sim 0.3 c$ ) whose mere existence has been a challenge to standard theoretical models of wind formation may well be responsible for the so-called AGN feedback and explain the AGN-host 
galaxy co-evolution (e.g., Tombesi et al. 2019; Laha et al. 2019, and references therein).

Remarkably, both phenomena (relativistic reflection and fast, massive outflows) are often seen together (Gallo et al. 2019; Walton et al. 2019). Consequently, understanding and disentangling their precise contribution has often been a matter of debate (Boller et al. 2002; Tanaka et al. 2004; Fabian et al. 2009; Miller et al. 2010; Zoghbi et al. 2011). This is because the emission and absorption features imprinted by partialcovering multiple-ionization absorbers can mimic, within available data, those expected from reflection components and vice versa (Done et al. 2007; Miller et al. 2009; Gallo \& Fabian 2011, 2013; Miller \& Turner 2013).

In order to shed light on these studies, a combination of highresolution spectra (to detect lines) and high throughput (to fill in all energy channels) must be achieved, such as that provided by the X-IFU instrument onboard Athena (Barret et al. 2018). It is therefore not surprising that understanding black hole accretion and black hole outflows are core science objectives for Athena (Nandra et al. 2013; Dovciak et al. 2013; Cappi et al. 2013). The $\mathrm{X}$-IFU combines a better-than $2.5 \mathrm{eV}$ spectral resolution up to $7 \mathrm{keV}$, and a peak effective area of $\sim 1 \mathrm{~m}^{2}$ at $1 \mathrm{keV}$, and capabilities to observe the brightest known AGNs with $~ 100 \%$ throughput (Barret et al. 2018).

Here we investigate for the first time the accuracy reached by $\mathrm{X}$-IFU observations in measuring black hole spins, the geometry of the reflection, and the parameters of the absorbers, considering realistic though complex multi-component AGN spectra. In what follows, we first present our methodology to model, simulate, and fit spectra (Sect. 2), present the results of a set of representative simulations highlighting some key parameters of the model (Sect. 3), describe the prospects of measuring UFOs in high-redshift objects (Sect. 4), and introduce a method to estimate systematic errors due to calibration errors (Sect. 5). The results are discussed in Sect. 6, where a comparison with similar studies is presented.

\section{Methodology}

\subsection{Model settings}

To simulate AGN X-IFU spectra, we assume an underlying continuum X-ray emission which consists of a hard cutoff power-law component and its relativistically smeared ionized reflection, assuming a lamp-post geometry for the irradiating source (relxilllp ${ }^{1}$ in XSPEC; Dauser et al. 2013; García et al. 2014). The free parameters of the model are the photon index $(\gamma)$ of the incident continuum, the height of the lamp post $(h$, in units of gravitational radius, $R_{\mathrm{g}}=G M / c^{2}$ ), the black hole spin parameter $(a)$, and the inclination ( Incl), ionization $(\log \xi)$, and iron abundance ( $A_{\mathrm{Fe}}$ in solar units) of the accretion disk. The high-energy cutoff of the power law is fixed to $300 \mathrm{keV}$, meaning that we do not have any curvature in the X-IFU energy range. When simulating the spectrum, the reflection fraction (reflfrac, hereafter $R_{\mathrm{f}}$ ) can be either forced to a specified value (setting the model parameter fixReflFrac to 0 ) or computed self-consistently within the model and fixed to the lamp-post value (setting fixReflFrac to 1), as defined in Dauser et al. (2016). The inner disk extends from the radius of the (spin dependent) innermost stable circular orbit up to 400 gravitational radii. The height of the compact source is constrained to lie within 3 and $10 R_{\mathrm{g}}$, consistent with the observations (Fabian et al. 2009; De Marco et al. 2013;

\footnotetext{
1 The model can be downloaded from http://www. sternwarte
} uni-erlangen . de/ dauser/research/relxill/
Emmanoulopoulos et al. 2014; Gallo et al. 2015). Here we consider only positive black hole spin values, limited to 0.998 .

The primary power law plus disk reflected emission are then seen through three absorbers of varying column density $\left(\mathrm{N}_{\mathrm{H} 1,2,3}\right)$, ionization parameter $\left(\log \xi_{1,2,3}\right)$, and covering factor $\left(c v f_{1,2,3}\right)$. The absorber thought to be closer to the black hole has a higher column density and higher ionization, and the other two have nonoverlapping but continuous $\mathrm{N}_{\mathrm{H}}$ and ionization parameters. Here we arbitrarily constrain the covering factor to range between 0.4 and 0.9. The absorber is modeled with zxipcf in XSPEC. For the absorption, zxipcf uses a grid of XSTAR photoionised absorption models (calculated assuming a micro-turbulent velocity of $200 \mathrm{~km} \mathrm{~s}^{-1}$, Kallman \& Bautista 2001; Reeves et al. 2008). Having the turbulent velocity as a free parameter of the model will soon be implemented (C. Done, priv. comm.).

Reflection on cold distant neutral material is also accounted for and is also subject to obscuration by the two most-distant, least-ionized absorbers. Cold reflection is modeled using the xillver model (García et al. 2013; but see e.g., Tanimoto et al. 2019 for a recent discussion on torus based models). Only the reflected component is computed setting refffrac $=-1$. The power law index of the irradiating source is tight to the one of the primary emission (with again the same high energy cutoff set to $300 \mathrm{keV}$ ). The iron abundance of the reflector is set to 1 and its ionization parameter is set to $1(\log \xi=0)$, meaning an almost neutral reflector. It is known that the resolution of the xillver model, currently $17 \mathrm{eV}$ at $6 \mathrm{keV}$ (García \& Kallman 2010), is significantly worse than the $2.5 \mathrm{eV}$ X-IFU spectral resolution. However, for the prime focus of this paper, this is unlikely to be an issue, as we are interested primarily in the relativistically smeared reflection component, with broadening exceeding the resolution of the model. Reflection models with finer resolution to fully exploit the X-IFU capabilities may become available (J. Garcia, priv. comm.).

We first consider a system inclination of $30^{\circ}$ and a redshift of all the components set to zero (unless stated otherwise). Galactic absorption is modeled through TBabs (Verner et al. 1993; Wilms et al. 2000), with $\mathrm{N}_{\mathrm{H}}$ being allowed to vary between 1 and $5 \times 10^{20} \mathrm{~cm}^{-2}$. In XSPEC terminology, the model considered is TBabs $\times\left(z x i p c f_{1} \times z_{\text {zip }} f_{2} \times\right.$ zxipc $_{3} \times$ relxilllp + zxipc $f_{1} \times$ zxipc $f_{2} \times$ xillver $)$. The main parameters of the model are presented in Table 1 together with their allowed range of variations. These values are estimated from de La Calle Pérez et al. (2010), Tombesi et al. (2010), Laha et al. (2014) and can be considered representative of typical values of nearby Seyfert 1 galaxies. An example of a simulated X-IFU spectrum highlighting the imprint of the various absorbers and the contribution of the different reflectors is shown in Fig. 1. Beside the forest of absorption lines present in the spectrum, it is worth noticing that the shape of the ionized reflection component shows multiple bumpy features below $\sim 2.5 \mathrm{keV}$ (see bottom left panel of Fig. 1), which is key in constraining the black hole spin, in support of the constraints provided by the broad iron line above $6 \mathrm{keV}$.

\subsection{Simulation setup and fitting}

The simulations are performed with the PyXspec interface to the XSPEC spectral-fitting program (Arnaud 1996). Here we use a built-in version of XSPEC 12.10.1. For simulations intended to sample the spin parameter space, we consider 50 regularly spaced values ranging from 0 to 0.995 . All other free physical model parameters are drawn from a uniform distribution bounded by their allowed interval of variations (listed in Table 1). The overall model is first normalized to correspond to an absorbed flux equivalent to a $1 \mathrm{mCrab}$ source in the $2-10 \mathrm{keV}$ 
Table 1. Model name, parameter, and range of values (between Min and Max) assumed in the simulations (see text for details).

\begin{tabular}{lccc}
\hline \hline Model & Parameter & Min & Max \\
\hline TBabs & $\mathrm{N}_{\mathrm{H}}$ & 0.01 & 0.05 \\
\hline \multirow{3}{*}{ xipc $f_{1}$} & $\mathrm{~N}_{\mathrm{H} 1}$ & 0.3 & 0.6 \\
& $\log \xi_{1}$ & 0.5 & 1.5 \\
& $c v f_{1}$ & 0.4 & 0.9 \\
\hline \multirow{3}{*}{ xipc $f_{2}$} & $\mathrm{~N}_{\mathrm{H} 2}$ & 0.6 & 1.0 \\
& $\log \xi_{2}$ & 1.5 & 3.0 \\
& $c v f_{2}$ & 0.4 & 0.9 \\
\hline \multirow{4}{*}{ xipcf $f_{3}$} & $\mathrm{~N}_{\mathrm{H} 3}$ & 5 & 10 \\
& $\log \xi_{3}$ & 2.5 & 4.0 \\
& $c v f_{3}$ & 0.4 & 0.9 \\
\hline & $a$ & 0.05 & 0.95 \\
& $h$ & 3.0 & 10.0 \\
relxilllp & $\gamma_{\text {relxillp }}$ & 1.7 & 2.2 \\
& $\log \xi$ & 2.0 & $\ldots$ \\
& $A_{\mathrm{Fe}}$ & 1.0 & 2.0 \\
& $\mathrm{Incl}$ & 30 & $\ldots$ \\
& $R_{\mathrm{f}}$ & $\ldots$ & 2.0 \\
\hline \multirow{6}{*}{ xillver } & $A_{\mathrm{Fe}}$ & 1.0 & $\ldots$ \\
& $\log \xi$ & 0 & $\ldots$ \\
& $\gamma$ & $\gamma_{\text {relxillp }}$ & $\ldots$ \\
& $\mathrm{Incl}$ & 30. & $\ldots$ \\
\hline
\end{tabular}

Notes. $\mathrm{N}_{\mathrm{H}}, \xi$, height $(h)$, and inclination (Incl) are given in units of $10^{22} \mathrm{~cm}^{-2}$, erg cm${ }^{-1} \mathrm{~s}, R_{\mathrm{g}}$, and degrees, respectively.

range (or an absorbed flux of $2 \times 10^{-11} \mathrm{ergs}^{2} \mathrm{~cm}^{-1}$ ). The sample size of 50 is commensurate with the number of known unabsorbed (typically type 1) AGN of similar brightness, which currently populate the Athena mock observing plan ${ }^{2}$, and can be found in common catalogs such as the 3XMM-DR8 (Rosen et al. 2019), Chandra (Evans et al. 2019), Swift (Oh et al. 2018) catalogs.

The normalization of the cold reflection xillver component is realistically assumed to be one fifth of the relxilllp component. As a sanity check of this assumption, we fitted the $2-10 \mathrm{keV}$ spectrum with a simple power-law model plus three Gaussian lines, and the best-fit equivalent width of the emission lines at $\sim 6.2,6.4$, and $7.1 \mathrm{keV}$ were 70,120 , and $20 \mathrm{eV}$, respectively. These values are broadly consistent with typical values measured for the FeK line redshifted, neutral, and $\mathrm{K}_{\beta}$ components (e.g. Guainazzi et al. 2006; Nandra et al. 2007; de La Calle Pérez et al. 2010). The spectra are then generated using the latest response matrices of the X-IFU (Barret et al. 2018) and the latest background files ${ }^{3}$. We note that the background rate for a point source with an extraction radius of $5^{\prime \prime}$ is less than $2 \times 10^{-4}$ counts s ${ }^{-1}$ and is negligible in the simulations (one mCrab source generates about 100 counts $\mathrm{s}^{-1}$ ). For grouping the spectral bins, we consider the optimal binning scheme of Kaastra \& Bleeker (2016) using the ftools ftgrouppha. The scheme accounts in particular for the energy-dependent spectral resolution of the instrument and the

\footnotetext{
2 The Athena mock observing plan can be downloaded from http://www.isdc. unige.ch/athena/document-repository/ category/192-general-interest.html

3 Available for download from http://x-ifu.irap.omp.eu/ resources-for-users-and- $\mathrm{x}$ - $\mathrm{ifu}$-consortium-members/.

The response files used here are named XIFU_CC_BASELINECONF_2018_10_10, which correspond to the configuration of the X-IFU presented at the Instrument Preliminary Requirement Review.
}

statistic of the spectrum (narrower bins near high-count regions and wider bins near low-count regions). Depending on the model parameter simulated (e.g., slope of the power law, absorber column density), the $1 \mathrm{mCrab}$ count rate varies from $\sim 50$ counts s$^{-1}$ to $\sim 120$ counts $\mathrm{s}^{-1}$ over the considered $\mathrm{X}$-IFU fitting energy range $(0.3 \mathrm{keV}-11.5 \mathrm{keV})$. When grouped, the mean energy bin width is less than $3 \mathrm{eV}$. There are 16 free parameters for the model considered here, and more than $6000^{\circ}$ of freedom (for a source of 1 mCrab brightness).

We use the so-called cstat metric in fitting the spectra (Cash 1979; Kaastra 2017b, see however Appendix A for an illustrative example of biases introduced by the use of the $\chi^{2}$ statistic). To fit a spectrum, as with real data we do not know what the model parameters will be, we do not initialize the fit with the input model parameters. Instead, we draw ten (up to 50) sets of randomly distributed parameters in their allowed interval of variations. For the normalization of the primary emission and cold reflection components, we draw two numbers from a uniform distribution bounded as $\pm 50 \%$ of the input normalizations. To speed up the fitting, we constrain the fit to converge in 50 iterations, with a critical change in the fit statistic $\Delta$ cstat $=0.1$, assuming that any better fit will be found during the error computation (on all free model parameters). As these starting parameters may be far off from the input spectral parameters, the fit may not reach an acceptable solution before its 50 iterations and is simply ignored (of the 50 initial sets of parameters, at least one set leads to an acceptable fit to launch the error computation). This method has the advantage that it sweeps all over the parameter space and reduces the chance that the minimization routine gets locked into a local minimum, away from the best fit.

For computing the errors on the best-fit parameters, we consider the set of best-fit parameters that provided the lowest cstat. For the parameter of interest, the positive and negative errors are computed by varying its value around its best-fit value, freezing it, and fitting the spectrum with all the other free parameters allowed to vary. The value is incremented until it exceeds a critical threshold $(\Delta \mathrm{cstat}=2.706$ for $90 \%$ confidence level errors). The parameter value at $\Delta$ cstat $=2.706$ is obtained through interpolation of the $\Delta$ cstat curve. This is equivalent to the recommended steppar procedure in XSPEC. If a new best fit is found along the error computation, the procedure aborts and then restarts on the first free parameter from the newly found best fit. Computing the errors further sweeps over the parameter space, and is often used to get away from local minima in the fitting statistics (e.g., Hurkett et al. 2008). With the method to initialize the fit described above, considering Simulation 1 described below, the mean decrease of cstat over 50 simulations along the error computation is $\sim 0.1$ (for a mean value of $\sim 6405$ ), indicating that the global minimum was likely found, hence the best fit. This is further supported by computing the goodness of the fit. Following Kaastra (2017b), we compute the goodness of the fit from the expected value and expected variance of the cstat. Again, in Simulation 1 described below, all measured cstat values for the best fits are within $\pm 2 \sigma$ of their expected values, indicating that the spectral model is acceptable. A visual inspection of the $\Delta$ cstat was applied to check on the behavior of some sensitive model parameters, such as the black hole spin.

\section{X-IFU spectral simulations in representative configurations}

In the simulations, we assume an effective integration time of $100 \mathrm{ks}$ (unless mentioned otherwise), with the implicit assumption that all model parameters remain constant within that 

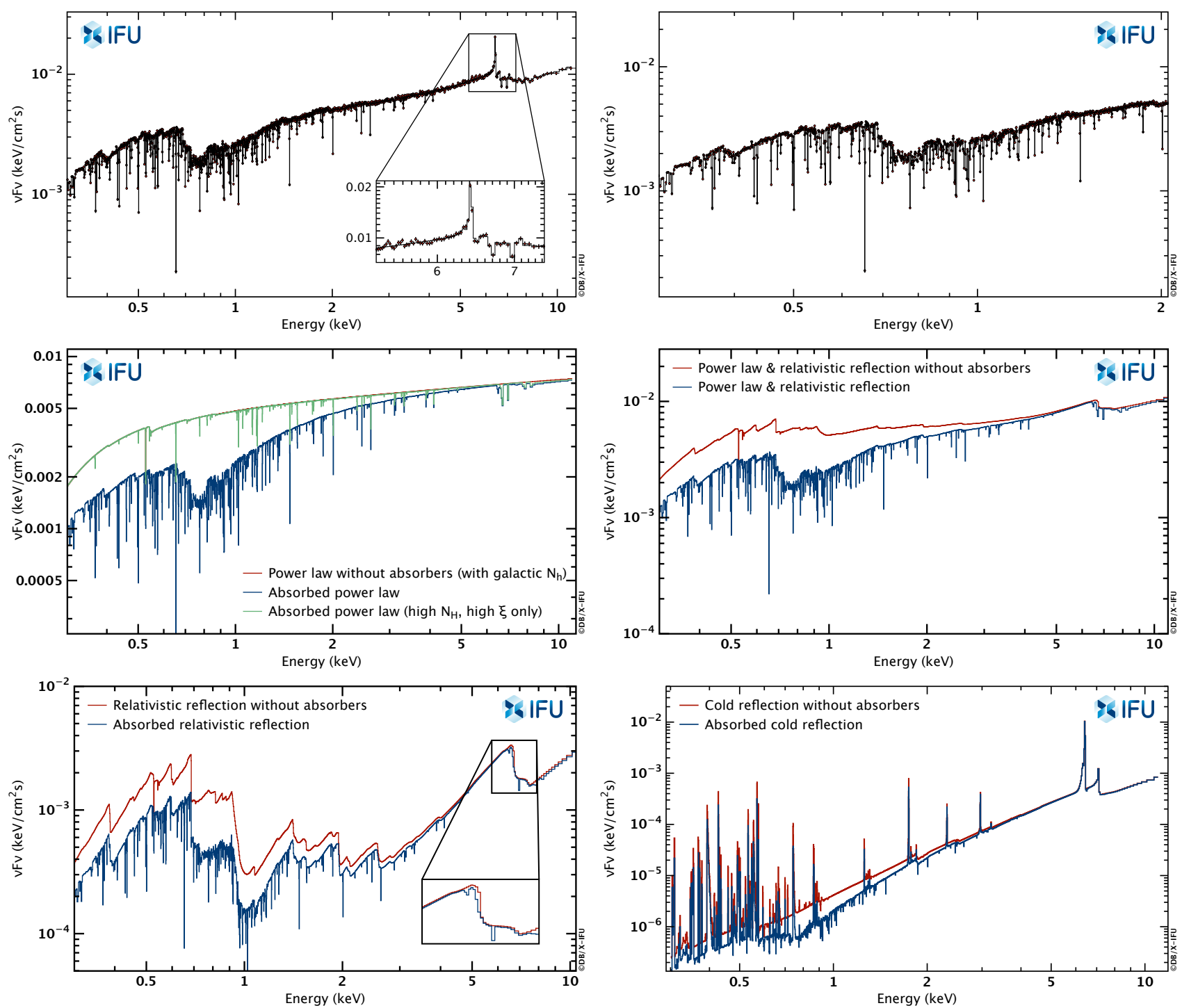

Fig. 1. Top-left: simulated X-IFU spectrum for a black hole spin parameter of 0.65 for a $R_{\mathrm{f}}=2, A_{\mathrm{Fe}}=2$ and $\log \xi=2$ with an inset around the FeK energy band. The source flux corresponds to a 1 mCrab source, and the integration time is set to $100 \mathrm{ks}$. Top-right: zoom of the spectrum below $2 \mathrm{keV}$. The imprint of the absorbers on the various broadband emission components is shown in the subsequent panels: Middle-left: power law. Middle-right: power law plus relativistic reflection component. Bottom-left: relativistic reflection component. Bottom-right: cold reflection component from distant material. The different components are shown with and without the absorbers. Bottom lower left panel: multiple bumpy features below $2 \mathrm{keV}$ due to ionized reflection. These are key in constraining the black hole spin.

duration (e.g., the height of the compact irradiating source, the parameters of the absorbers, etc.).

\subsection{The most conservative case: $R_{\mathrm{f}}=1, A_{\mathrm{Fe}}=1$ for $a$ 1 mCrab source (configuration 1)}

To demonstrate the power of the X-IFU to constrain black hole spins, the very first simulation to be conducted assumes the most conservative case, in which the iron abundance and the reflection fraction are both set to a value of 1 , and we consider a mildly ionized disk with the ionization parameter set to $\log \xi=2$. We assume a $1 \mathrm{mCrab}$ source. We simulate 50 spectra with positive spins ranging from 0 to 0.995 in regular spacing, to make sure that the whole spin range is covered. The mean error on the best fit parameters for the three absorbers and the reflection component are listed in Table 2. As can be seen, the statistical error on the spin parameter is on average $\leq 0.1$, and the error on the height of the irradiating source is $\sim 0.3 R_{\mathrm{g}}$.

\subsection{Another conservative case: fixed lamp-post geometry with $A_{\mathrm{Fe}}=1$ for a $1 \mathrm{mCrab}$ source (configuration 2)}

In this simulation run, the parameters of the reflection component are computed by the model to the predicted value of the current parameter configuration in the lamp-post geometry (Dauser et al. 2016), namely the height of the irradiating source and the spin. The parameter $R_{\mathrm{f}}$ is then left free to vary in the fit. The iron abundance is conservatively set to one and the ionization parameter of the accretion disk remains set to two. We again consider 50 spectra with positive spins ranging from 0 to 0.995 with regular spacing, while for each spin, $h$ is drawn from a uniform distribution between 3 and $10 R_{\mathrm{g}}$. The mean error on the best fit parameters for the three absorbers and the reflection component are listed in Table 2 . The best-fit parameters $\left(a, h, R_{\mathrm{f}}\right)$ are shown in Fig. 2. The reflection fraction computed from the model is everywhere smaller than 2 , but larger than in the most conservative case discussed above; it varies from $\sim 1.2$ for low spin values up to $\sim 1.9$ at the highest spins. The higher reflection 
D. Barret and M. Cappi: High resolution X-ray spectroscopy of accreting supermassive black holes

Table 2. Mean 90\% confidence level one-sided errors from the best-fit spectral parameters of the various configurations of the simulations.

\begin{tabular}{cccccccccccccc}
\hline \hline Conf. & $\Delta \mathrm{N}_{\mathrm{H} 1}$ & $\Delta \log \xi_{1}$ & $\Delta c v f_{1}$ & $\Delta \mathrm{N}_{\mathrm{H} 2}$ & $\Delta \log \xi_{2}$ & $\Delta c v f_{2}$ & $\Delta \mathrm{N}_{\mathrm{H} 3}$ & $\Delta \log \xi_{3}$ & $\Delta c v f_{3}$ & $\Delta h$ & $\Delta a$ & $\Delta \gamma$ & $\Delta R_{\mathrm{f}}$ \\
\hline 1 & 0.011 & 0.013 & 0.010 & 0.052 & 0.036 & 0.032 & 0.498 & 0.024 & 0.027 & 0.296 & 0.083 & 0.003 & 0.027 \\
$1 \mathrm{~b}$ & 0.044 & 0.053 & 0.049 & 0.164 & 0.074 & 0.081 & 1.579 & 0.063 & 0.097 & 0.592 & 0.148 & 0.004 & 0.042 \\
2 & 0.012 & 0.014 & 0.012 & 0.056 & 0.036 & 0.034 & 0.475 & 0.022 & 0.023 & 0.217 & 0.048 & 0.003 & 0.036 \\
3 & 0.013 & 0.018 & 0.015 & 0.058 & 0.036 & 0.034 & 0.595 & 0.027 & 0.026 & 0.178 & 0.049 & 0.003 & 0.038 \\
3b & 0.016 & 0.022 & 0.019 & 0.065 & 0.035 & 0.030 & 0.521 & 0.021 & 0.023 & 0.180 & 0.045 & 0.003 & 0.042 \\
4 & 0.043 & 0.050 & 0.040 & 0.174 & 0.086 & 0.077 & 1.574 & 0.066 & 0.068 & 0.595 & 0.168 & 0.009 & 0.119 \\
5 & 0.043 & 0.049 & 0.036 & 0.169 & 0.076 & 0.068 & 1.506 & 0.066 & 0.078 & 0.610 & $\ldots$ & 0.009 & 0.066 \\
6 & 0.041 & 0.044 & 0.035 & 0.112 & 0.061 & 0.055 & 2.162 & 0.077 & 0.082 & 0.604 & $\ldots$ & 0.008 & 0.063 \\
7 & 0.027 & 0.028 & 0.026 & 0.090 & 0.045 & 0.040 & 0.753 & 0.031 & 0.031 & 0.495 & 0.113 & 0.004 & 0.070 \\
8 & 0.027 & 0.027 & 0.030 & 0.074 & 0.045 & 0.046 & 0.767 & 0.044 & 0.041 & 0.543 & 0.107 & 0.004 & 0.051 \\
9 & 0.021 & 0.025 & 0.017 & 0.063 & 0.037 & 0.028 & 0.660 & 0.026 & 0.029 & 0.370 & 0.118 & 0.005 & 0.054 \\
\hline
\end{tabular}

Notes. Only the parameters of the three absorbers and those of the reflection component are listed. The mean one-sided errors are the mean of the positive and negative errors. We note that the positive error on the spin is bounded by the maximum spin value of 0.998 . Configuration $1 \mathrm{~b}$ corresponds to the same simulations as for configuration 1 but using the WFI responses (Meidinger et al. 2018). Configuration $3 \mathrm{~b}$ is identical to configuration 3 but a 5\% energy-independent systematic uncertainty has been added to the data. The integration time for configurations 1 to 4 , and 7 to 9 is $100 \mathrm{ks}$; for configurations 5 and 6 , this is $25 \mathrm{ks}$. The source intensity is $1 \mathrm{mCrab}$ in configurations 1 to 4 and $0.5 \mathrm{mCrab}$ in configurations 5 to 9 . Configurations 7 to 9 are identical to configuration 3 but the iron abundance, the system inclination, and the disk ionization are allowed to vary, as indicated in Table 3 .
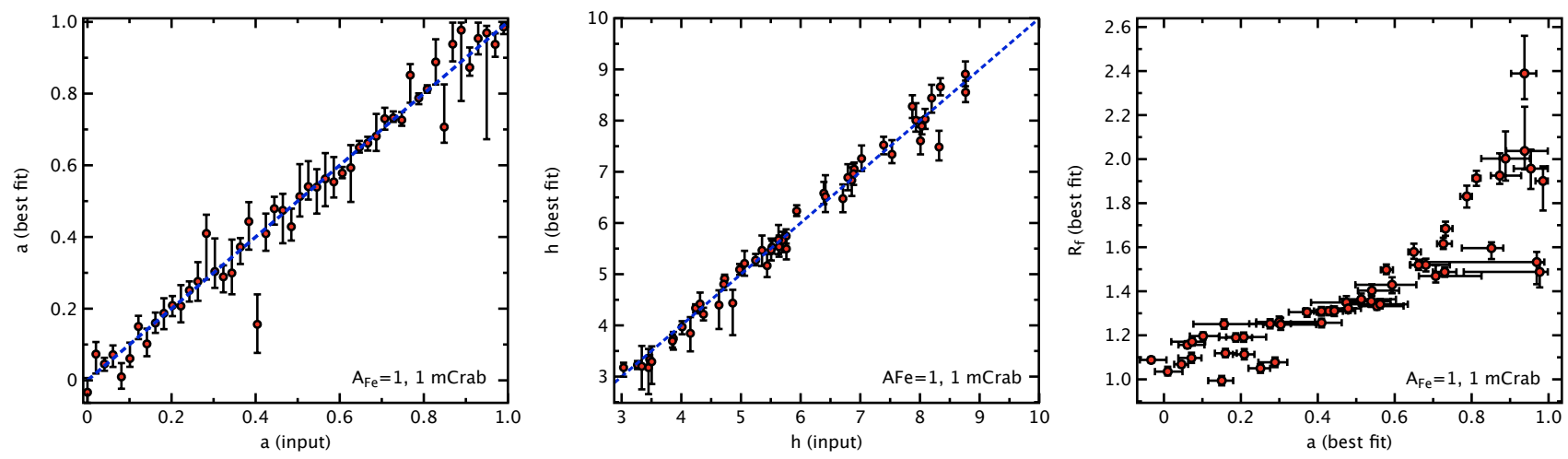

Fig. 2. Best fit parameters vs. input parameters for the case of a $1 \mathrm{mCrab}$ source observed for a $100 \mathrm{ks} \mathrm{X}$-IFU observation. The parameters of the reflection component are computed by the model to the predicted value of the geometrical configuration in the lamp-post geometry (Dauser et al. 2016) and then left as a free parameter of the fit. Fifty spins are generated to sample the range $0-0.995$, while the height of the X-ray source is drawn at each spin from a uniform distribution bounded between 3 and $10 R_{\mathrm{g}}$. The iron abundance $A_{\mathrm{Fe}}$ is set to 1 . The simulations are performed in the so-called configuration 2 described in Sect. 3.2. Left: spin parameter. Middle: height of the compact source. Right: reflection fraction vs. fitted spin parameters. The errors are computed at the $90 \%$ confidence level for variation of one single parameter. As predicted by the model, $R_{\mathrm{f}}$ tends to increase with the black hole spin; the scattering at high spins is due to the height of the X-ray source being allowed to vary between each simulation run.

fraction at high spins compensates for the increased smearing of the reflection features, likely explaining why the error bars on the spin remain similar across the spin range. As listed in Table 2 the accuracy of the fitted spin values has a mean error of $\sim 0.05$ across the spin range considered, while the height of the irradiating source is accurate to $\sim 0.2 R_{\mathrm{g}}$.

\subsection{Setting $R_{\mathrm{f}}=2, A_{\mathrm{Fe}}=2$ for a $1 \mathrm{mCrab}$ source (configuration 3 )}

Although its physical origin has often been debated (but see the hypothesis on radiative levitation by Reynolds et al. (2012), iron overabundance and large $R_{\mathrm{f}}$ have often been reported from AGN $\mathrm{X}$-ray spectra, with values reaching 10 and 5, respectively, in the most extreme cases (and references therein Fabian et al. 2009; Risaliti et al. 2013; Parker et al. 2018b). Similarly, iron overabundance is also inferred from fitting binary black hole spectra (García et al. 2018), with values several times solar being rou- tinely found. Here we assume a reflection fraction of two and iron overabundance by a factor of two at maximum, which may not be considered such an extreme case after all. $R_{\mathrm{f}}$ is fixed in faking the spectra, and then left as a free parameter of the fit. Fifty sets of the remaining 15 parameters are drawn from their uniform distribution, with the spin ranging from 0 to 0.995 with regular spacing. The mean error on the best-fit parameters for the three absorbers and the reflection component are listed in Table 2. The best-fit parameters against the input values of the model are presented in Fig. 3. There are several noticeable features in this figure. First the spin parameter is very well constrained, and as expected, as the smearing increases with the spin, the recovered error on the spin increases towards the highest spin values (this is not compensated by a larger $R_{\mathrm{f}}$ as it is fixed to a value of 2 in the simulations). Nevertheless, the mean error on the spin parameter is $\lesssim 0.05$ across the range of spins considered. Second, in the lamp-post geometry, the height of the compact source is also very well constrained with a mean error 

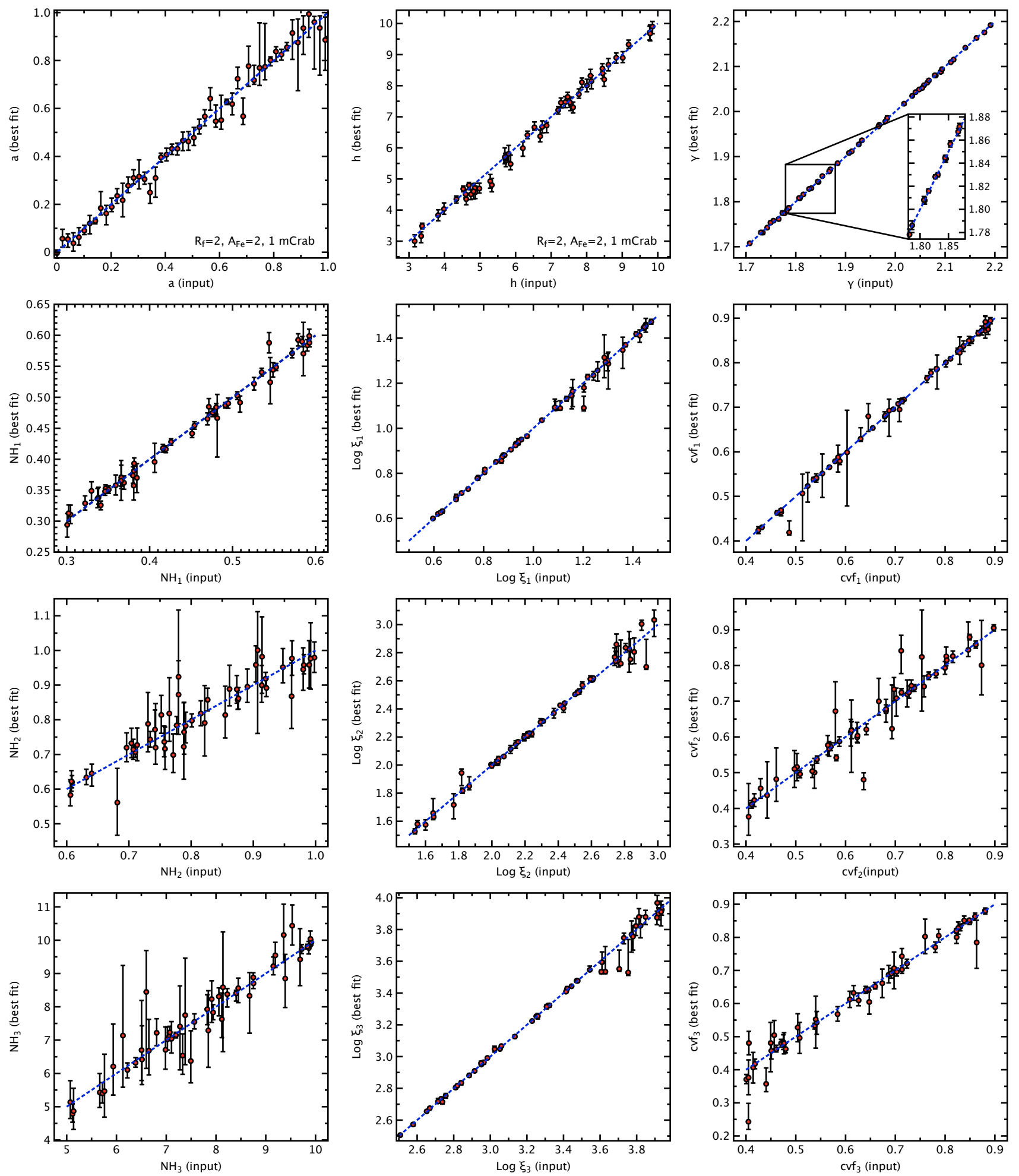

Fig. 3. Best-fit parameters vs. input parameters for the simulations of a $1 \mathrm{mCrab}$ source observed for a $100 \mathrm{ks} \mathrm{X}$-IFU observation $\left(R_{\mathrm{f}}=2, A_{\mathrm{Fe}}=2\right.$, $1 \mathrm{mCrab}, 100 \mathrm{ks}$, so-called configuration 3). Fifty spins are generated with a constant spacing between 0 and 0.995 . All other parameters are drawn from uniform distributions within bounds listed in Table 1. Top-left: black hole spin. Top-middle: height of the coronal source. Top-right: photon index of its spectrum. Then downwards, from left-to-right: parameters of the three absorbers: $\mathrm{N}_{\mathrm{H}}$, the ionization (log $\xi$ ), and the covering factor. Errors are computed at the $90 \%$ confidence level for variation of one single parameter. The mean errors for the spin and height of the irradiating source are $\sim 0.05$ and $\sim 0.18 R_{\mathrm{g}}$ respectively. The mean errors for all parameters are listed in Table 2 . In particular, the power-law index is accurately determined and shows no bias (see Appendix A).

of the order of $\sim 0.2 R_{\mathrm{g}}$. The parameter $R_{\mathrm{f}}$ as recovered by the fit is shown in Fig. 4; it is again determined with an accuracy of $\sim 2 \%$. Similarly, the power-law index of the hard irradiating source has a negligible error (0.003), showing no bias against its input value (see Appendix A for the bias that would be introduced by using $\chi^{2}$ as the fitting metric). Finally, the parameters 


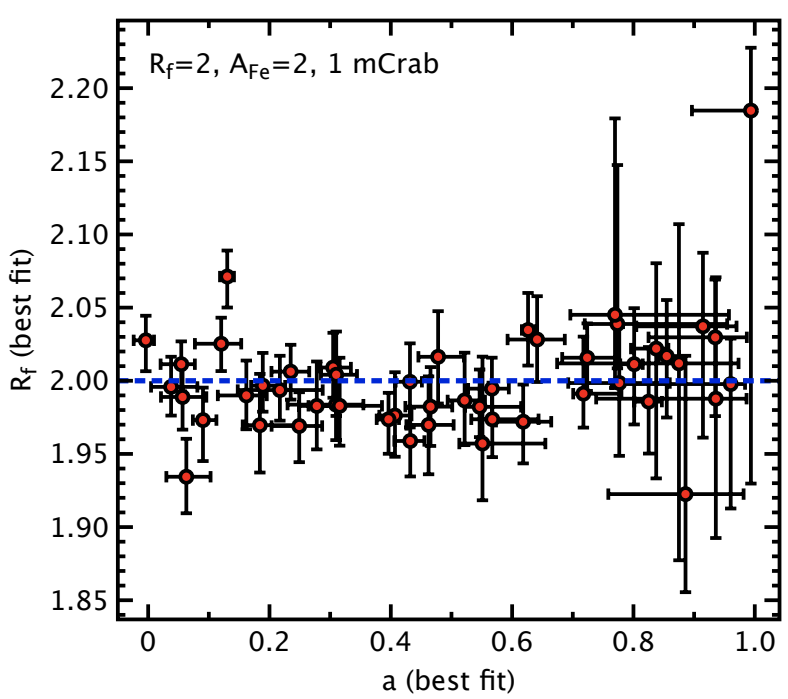

Fig. 4. Reflection fraction recovered from the fit of configuration 3 spectra $\left(R_{\mathrm{f}}=2, A_{\mathrm{Fe}}=2,1 \mathrm{mCrab}\right)$. This figure complements Fig. 3 .

of the three absorber components are very well recovered, most notably the ionization parameter (typical errors of $\sim 2 \%$ over their range of variation). As expected at the highest ionization, the best-fit error increases as there are less lines in the spectra to hook the fit. A degeneracy between the $\mathrm{N}_{\mathrm{H}}$ and the covering factor may be expected (a higher $\mathrm{N}_{\mathrm{H}}$ and a smaller covering factor can be found as equal to a smaller $\mathrm{N}_{\mathrm{H}}$ and a higher covering factor). Despite this, the recovered values are all consistent or very close to their input values, demonstrating the power of high resolution and high throughput spectroscopy in deciphering multiple narrow and broad components in complex AGN spectra. Although not shown on this summary plot, it should be added that the normalization of the two reflection components and the galactic $\mathrm{N}_{\mathrm{H}}$ are also consistent with their input values.

\subsection{Setting $R_{\mathrm{f}}=2, A_{\mathrm{Fe}}=2$ for a $0.1 \mathrm{mCrab}$ source (configuration 4)}

Given the small uncertainties on the spin determination in Fig. 3, it is tempting to investigate how the X-IFU would perform on sources that are ten times weaker, opening the possibility to explore the spin distribution of weaker seyfert galaxies and/or more distant quasars. We therefore repeated the simulations above, but this time assuming a source corresponding to a flux of $0.1 \mathrm{mCrab}$, equivalent to $2 \times 10^{-12} \mathrm{ergs} \mathrm{cm}^{-2} \mathrm{~s}^{-1}$ $(2-10 \mathrm{keV})$, allowing the integration time to increase to $150 \mathrm{ks}$ (to compensate partly for the ten-times-lower brightness). The mean error on the best-fit parameters for the three absorbers and the reflection component are listed in Table 2. The results of the simulations are shown in Fig. 5 for a sample of 20 simulations. As can be seen, the spin determination is less accurate, yet the error bars on the spin parameter are typically $\sim 0.17$, with the same tendency as above for lower spin values to be determined more accurately, as expected given the sharper line features there.

\subsection{Recovering the height of the compact source on shorter timescales (configuration 5)}

Assuming that the spin of the black hole is known, it is worth investigating how the height of the compact source could be measured on timescales of the order of $25 \mathrm{ks}$, thought to be commensurable to the characteristic variability timescale of these sources, that is, typically bright nearby Seyfert galaxies (Ponti et al. 2012). We consider here a $0.5 \mathrm{mCrab}$ source with a spin parameter of 0.5 . We assume a conservative iron abundance for the disk, an ionization $\log \xi=2$, and $R_{\mathrm{f}}$ computed self-consistently from the relxill model in the lamp-post geometry, allowing the height of the irradiating source to vary between 3 and $10 R_{\mathrm{g}}$. We simulate $25 \mathrm{X}$-IFU spectra, with different corona heights, power-law index, and absorber parameters. We set the reflection fraction as a free parameter of the fit. The mean error on the best-fit parameters for the three absorbers and the reflection component are listed in Table 2. The best fit results for the compact source height and the reflection fraction are shown in Fig. 6. On a timescale of $25 \mathrm{ks}$, the accuracy with which $h$ can be measured is about $0.6 R_{\mathrm{g}}$, while $R_{\mathrm{f}}$ is determined with an accuracy of $5 \%$. Combining such spectral information with a timing analysis (e.g., measuring time lags) would enable a detailed mapping of the accretion geometry around the black hole.

\subsection{Recovering the parameters of the UFOs (configuration 6)}

We have previously demonstrated the capability of X-IFU to separate the three absorbers imprinting on a complex reflection spectrum. Next we focus on the third high-density, high ionization absorber when blueshifted. In low-resolution AGN $\mathrm{X}$-ray spectra, these absorbers manifest as narrow Fe $\mathrm{K}$-shell blueshifted absorption lines from Fe XXV/XXVI, with inferred radial velocities between 0.03 and $0.3 c$ (Tombesi et al. 2010; Gofford et al. 2013). As the blueshift increases, the strongest high-energy absorption lines due to iron get shifted towards higher energies and separate clearly from the relativistic iron line, but fall in an energy range where the effective area of the XIFU decreases sharply. Yet, as shown below, constraints on the parameters of this absorber are expected to come also from the low-energy absorption lines, which are also well resolved by the X-IFU.

We carry out a set of simulations, considering a fixed black hole spin (0.5), with reflection computed in a fixed lamp-post configuration, blueshifting the third absorber component with velocities ranging between -0.3 and -0.05 the speed of light, and the parameters of the three absorbers again drawn from uniform distributions bounded in their interval of variations listed in Table 1 (the redshift of the first two other absorbers remains at zero). We keep the same zxipcf model for the absorber, but we smear it with a Gaussian (gsmooth in XSPEC) to account for an additional broadening of $1000 \mathrm{~km} \mathrm{~s}^{-1}$ at $6 \mathrm{keV}$, consistent with UFO observations (Tombesi et al. 2011). This will smear out the absorption features, thus reducing the benefit of a highresolution spectrometer for that component, while it remains crucial to separate the other absorbers. The XSPEC model used is TBabs $\times\left(z x i p c f_{1} \times z x i p c f_{2} \times\left(z x i p c f_{3} \otimes\right.\right.$ gsmooth $) \times$ relxilll $p+$ zxipc $f_{1} \times$ zxipc $_{2} \times$ xillver $)$.

We assume a $0.5 \mathrm{mCrab}$ source and $25 \mathrm{ks}$ for the spectrum integration time, again because it is interesting to probe those UFOs on the shortest possible timescales. The mean error on the best-fit parameters for the three absorbers and the reflection component are listed in Table 2. The results of the fit for the case of a turbulent velocity of $1000 \mathrm{~km} \mathrm{~s}^{-1}$ are shown in Fig. 7. As can be seen, the accuracy by which the absorber parameters are recovered has decreased due to the smearing, although the redshift (i.e., velocity) of the absorber is measured with a very high accuracy. 

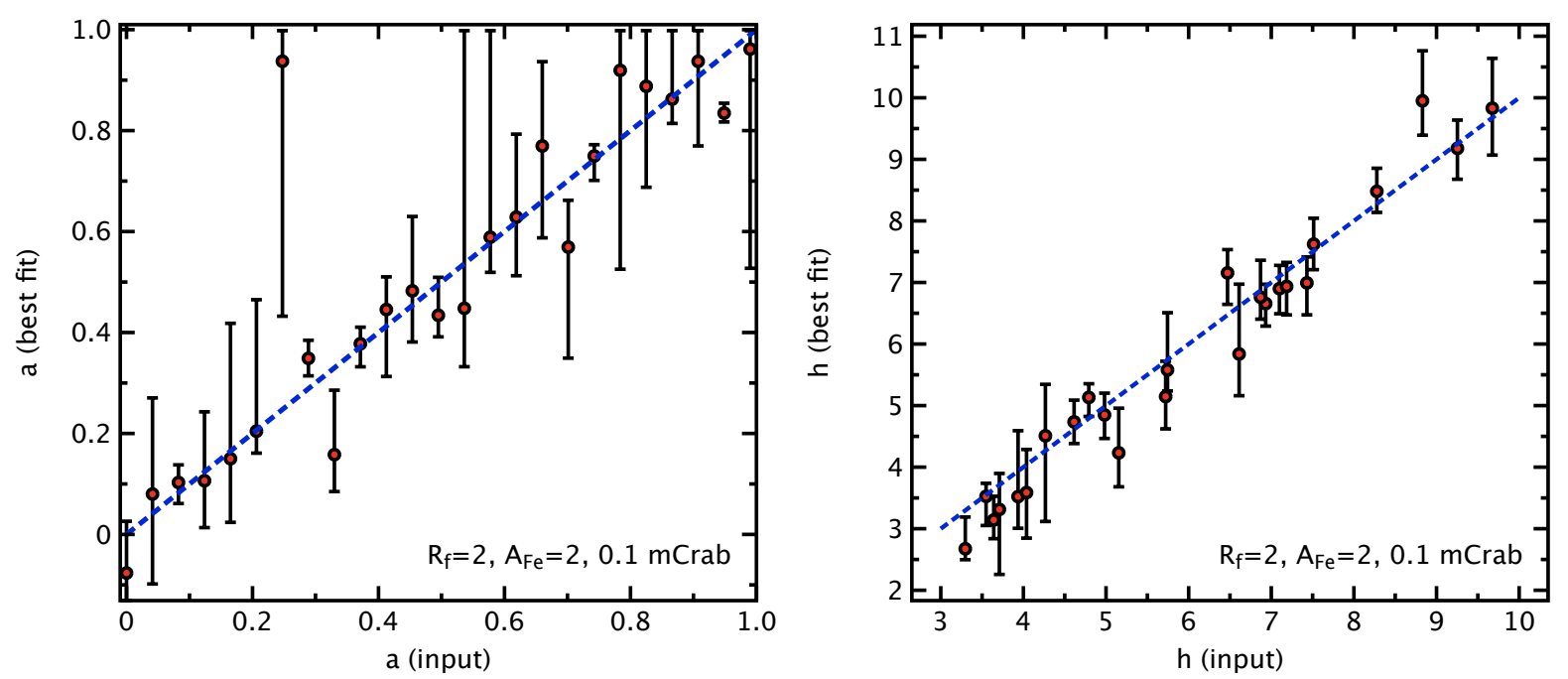

Fig. 5. Best-fit parameters vs. input parameters for the case of a 0.1 mCrab source observed for a $150 \mathrm{ks} X-I F U$ observation (configuration 4 , $R_{\mathrm{f}}=2, A_{\mathrm{Fe}}=2$ ). Fifty spins are generated with a constant spacing between 0 and 0.995 . The other parameters are allowed to vary with the range listed in Table 1. Left: black hole spin. Right: height of irradiating source against input values. Errors are computed at the $90 \%$ confidence level for variation of one single parameter. The mean errors on the spin and height of the irradiating source are $\sim 0.17$ and $\sim 0.6 R_{\mathrm{g}}$ respectively. The errors on all parameters are listed in Table 2.
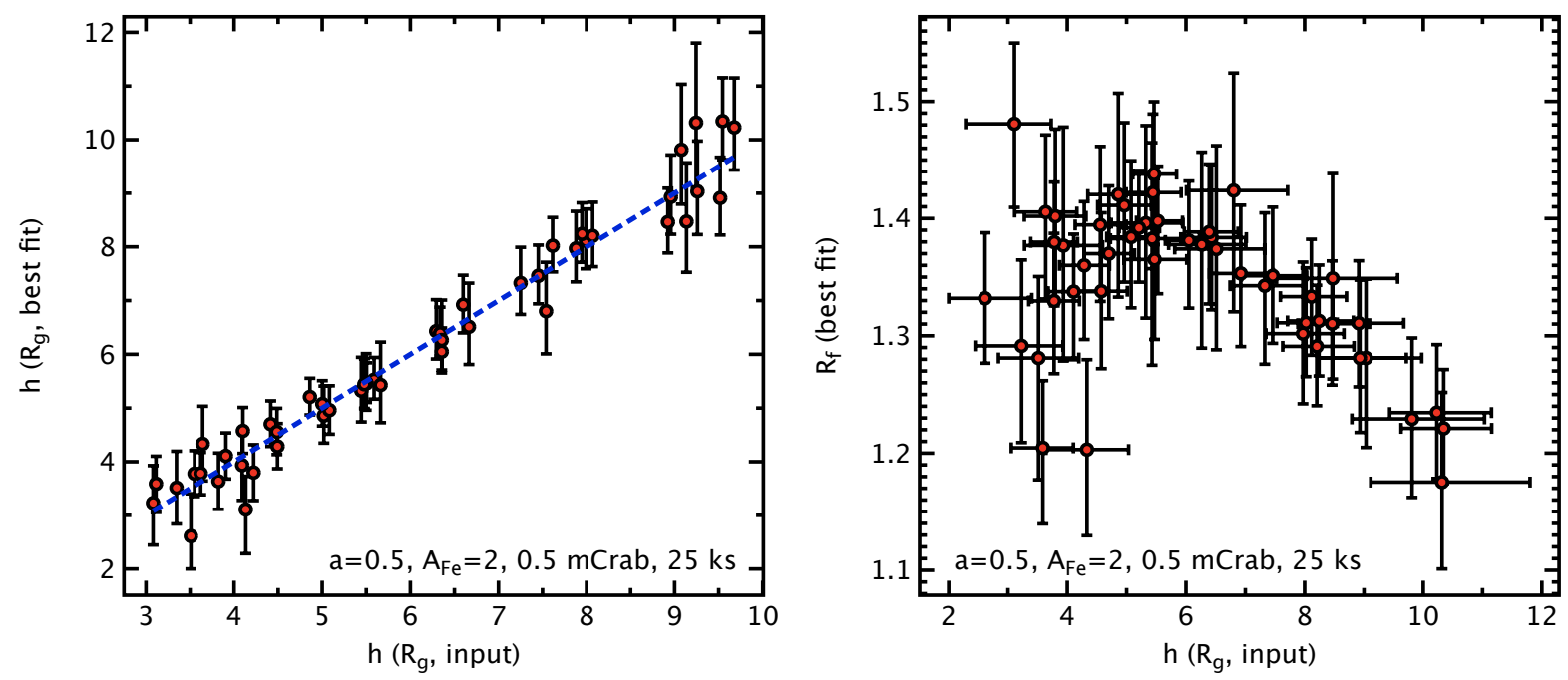

Fig. 6. Best-fit parameters vs. input parameters for the case of a $0.5 \mathrm{mCrab}$ source observed for a $25 \mathrm{ks} \mathrm{X}$-IFU observation (configuration 5 ). The spin is fixed to 0.5 and the other parameters are allowed to vary with the range listed in Table 1 . From left to right, the height of the coronal source against its input values, and the reflection fraction computed by the relxill model against the height of the coronal source. Errors are computed at the $90 \%$ confidence level for variation of one single parameter. As predicted by the model, $R_{\mathrm{f}}$ decreases with the height of the X-ray source. The mean error on $h$ is about $0.6 R_{\mathrm{g}}$.

\subsection{Varying other key parameters: iron abundance, system inclination, disk ionization (configurations 7 to 9)}

The above simulations have all considered fixed iron abundance $\left(A_{\mathrm{Fe}}=1\right.$ or 2$)$, fixed inclination $\left(30^{\circ}\right)$, and fixed ionization $(\log \xi=2)$ of the disk. It is interesting to see how those parameters can be constrained if they are left free to vary in the model, and what impact this would have on the spin measurement accuracy. We have simulated three sets of 25 spectra for a $0.5 \mathrm{mCrab}$ source, the spin covering 0 to 0.995 and an exposure time of $100 \mathrm{ks}\left(R_{\mathrm{f}}=2, A_{\mathrm{Fe}}=2\right)$. The range of allowed variations for $A_{\mathrm{Fe}}$, system inclination, and disk ionization are listed in Table 3. The mean error on these specific parameters is given as the last column of the table. The mean error on the other best-fit parameters is reported in Table 2 in the lines corresponding to configurations 7 to 9 . As can be seen, those parameters are recov- ered with high accuracy, most notably the varying disk ionization parameter which is one of the key parameters defining the reflection spectrum. Leaving those parameters free to vary in the fit degrades the accuracy by which the spin and the height of the irradiating source are measured by about a factor of two.

\subsection{Soft excess}

The measurement of the reflection parameters relies on the broadband coverage of the X-IFU; not only the iron $\mathrm{K} \alpha$ line $(6-7 \mathrm{keV})$, but also the relativistically smeared features below $2 \mathrm{keV}$ or so (see Fig. 1, bottom left panel). As a matter of fact, a simple test of ignoring the data below $2 \mathrm{keV}$ in the fit shows that it would significantly reduce the accuracy on the best-fit parameters for the complex model considered here. Below $2 \mathrm{keV}$ is an energy range 

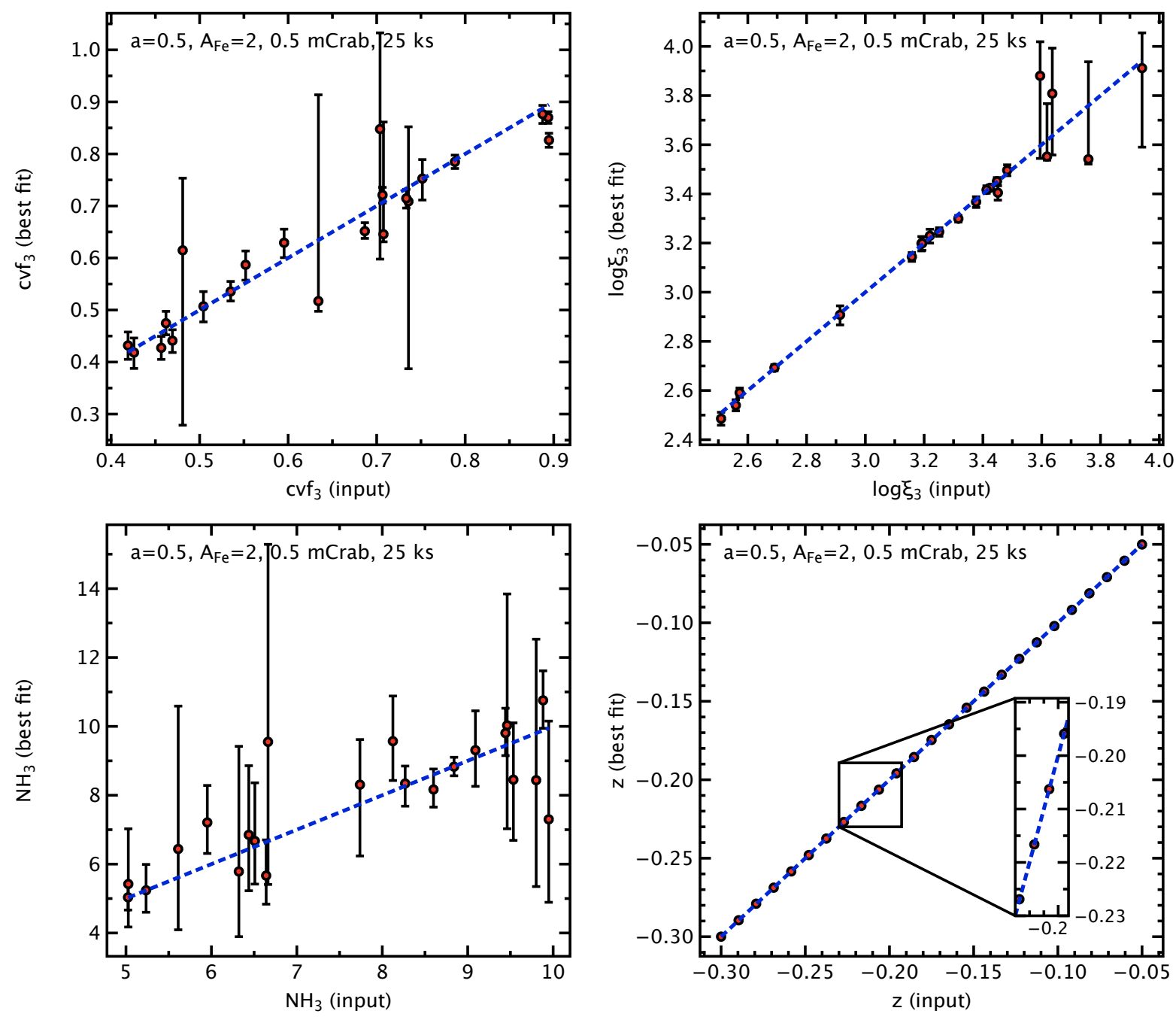

Fig. 7. Parameters of the high-velocity absorber measured for an integration time of $25 \mathrm{ks}$ for a $0.5 \mathrm{mCrab}$ source (configuration 6). The absorber is smeared with a broadening velocity of $1000 \mathrm{~km} \mathrm{~s}$ (at $6 \mathrm{keV}$; see text for details). As can be seen, the redshift of the absorber is measured with very high accuracy.

in which a soft excess (in addition to the underlying power-law component) is often requested by the data, with a significant contribution to the total flux. Its origin is still debated. Hypotheses include an extra warm Comptonization, complex partial covering, disk blackbody emission, a reprocessed reflection component, and even a relativistically blurred high-density reflection (Magdziarz et al. 1998; Crummy et al. 2006; Gierliński \& Done 2006; Mehdipour et al. 2015; Petrucci et al. 2018; Middei et al. 2019; García et al. 2019b). In addition to the fact that X-IFU with its unprecedented sensitivity at $\sim 1 \mathrm{keV}$ will provide critical insight into the origin of the soft excess, for this paper it is important to test whether the presence of such a soft component, if not related to relativistic reflection, could affect the accuracy by which the reflection parameters and the black hole spin are measured.

We have thus simulated a set of 50 spectra in configuration $3\left(R_{\mathrm{f}}=2, A_{\mathrm{Fe}}=2, \log \xi=2\right)$, adding to the input model a steep power law with a photon index ranging between 2.5 and 3.5. Alternative models for the soft excess would be a thermal comptonization, a comptt-like, or a blackbody model. The exact shape of the soft excess is of little importance here: what is important is the number of counts added on top of the reflection spectrum. The corresponding XSPEC model is then TBabs $\times\left(z x i p c f_{1} \times z x i p c f_{2} \times z x i p c f_{3} \times(\right.$ relxilllp + powerlaw $)+$ zxipc $\left.f_{1} \times z x i p c f_{2} \times x i l l v e r\right)$. The power law normalization is such that the $0.5-2 \mathrm{keV}$ flux of the power law component is conservatively set to $50 \%$ of the total flux in that energy range. This can be considered conservative as on average it leads to a $0.5-2 \mathrm{keV}$ unabsorbed flux higher than the $2-10 \mathrm{keV}$ flux (by $\sim 10 \%$ ), while they are generally found to be comparable (Miniutti et al. 2009). As we are only interested in the errors on the spin and height, the fit starts with the input model parameters and the errors are computed on these two parameters only. We find a mean error of $\sim 0.13$ on the spin and $\sim 0.36 R_{\mathrm{g}}$ on the height. This is to be compared with the values of $\sim 0.05$ and $\sim 0.18$, respectively, reported in Table 2. As expected, the accuracy on the spin and height measurement has decreased, because the broad features of the relativistic reflection below $2 \mathrm{keV}$ are diluted by the soft excess. They remain acceptable however in the conservative setting used for the model.

\section{Beyond the local universe: $a z=2.5$ AGN}

We have demonstrated above that even for moderately bright sources $(0.1 \mathrm{mCrab}) \mathrm{X}$-IFU is able to characterize both the absorption components and the reflection spectra simultaneously and with great precision. We now want to investigate how well it could perform on even more distant (i.e., fainter) sources, considering that significantly redshifting the spectrum would bring 
Table 3. Allowed range of variations for the iron abundance, system inclination, and the ionization parameter of the disk.

\begin{tabular}{clcc}
\hline \hline Configuration & Parameter & Range & Mean error \\
\hline 7 & $A_{\mathrm{Fe}}$ & $1-10$ & 0.269 \\
8 & Inclination (deg.) & $10-70$ & 0.123 \\
9 & $\log \xi$ & $1-3$ & 0.015 \\
\hline
\end{tabular}

Notes. The mean one-sided $90 \%$ errors on these parameters are listed from the fitting of 25 simulated spectra. Each simulation considers a $0.5 \mathrm{mCrab}$ source observed for $100 \mathrm{ks}$, a reflection fraction of two, and a uniform distribution of the varying parameter in their allowed range of variations. The errors on the other parameters are listed in Table 2 , including errors on the spin and the height of the irradiating source.

the absorption and emission features closer to the peak of the effective area of the X-IFU (see Fig. 10), thus partly compensating the reduction of flux.

The model we consider is a simplification of the model above in which the first two absorbers are merged into one. The XSPEC model becomes TBabs $\times\left(\right.$ zxipc $f_{1} \times\left(z x i p c f_{2} \otimes\right.$ gsmooth $) \times$ relxilllp $+z x i p c f_{1} \times$ xillver $)$. The covering factor of the two absorbers is set to an intermediate value of 0.75 . We therefore first consider an AGN with a flux of $2 \times 10^{-13} \mathrm{ergs} \mathrm{cm}^{-2} \mathrm{~s}^{-1}$ (i.e., 0.01 mCrab; Georgakakis et al. 2013; Martocchia et al. 2017; Dadina et al. 2018; Baronchelli et al. 2018). The normalization of the cold reflection component in comparison to the relativistic one remains one fifth. The reflection component is computed in a fixed lamp-post configuration assuming a black hole spin of 0.5 . We note that no meaningful constraints can be derived on the spin at this flux level. We further assume a height of the irradiating source of $4 R_{\mathrm{g}}$ with $A_{\mathrm{Fe}}=1$ and $\log \xi=2$. The redshift of the first absorber is set to the redshift of the source, while a blueshift (between -0.3 and -0.05 ) is added to the second absorber. A Gaussian velocity broadening of $1000 \mathrm{~km} \mathrm{~s}^{-1}$ normalized at $6 \mathrm{keV}$ (rest frame; the index of the Gaussian smoothing function in XSPEC is assumed to be 1) is assumed for the high-density absorber. A simulated spectrum corresponding to an exposure time of $100 \mathrm{ks}$ is shown in Fig. 8 to highlight the imprints of the two absorbers on the spectrum, leaving a forest of absorption lines that will be crucial to measure the redshifts.

To be more quantitative, we simulate ten spectra with the redshift of the source being allowed to vary between 2.4 and 2.6, and a large velocity broadening of $3000 \mathrm{~km} \mathrm{~s}^{-1}$ for the high-density absorbers. The fit is performed between 0.3 and $3.5 \mathrm{keV}$. Both the source redshift and the redshift of the absorber are then left free in the fit. In the framework of this simplistic model, unsurprisingly the redshift of the source would be determined with high accuracy due to the prominent redshifted iron line produced by the distant reflector (statistical error less than $\sim 0.001$ ). Despite the larger velocity broadening, the blueshift of the high-density absorber would be measured with a statistical error of much less than $\sim 0.01$. The best-fit X-ray redshifts for the source and the UFO are plotted in Fig. 9. More detailed simulations are warranted with added complexity to the model, but as discussed by Martocchia et al. (2017), who pushed the flux limit by yet another order of magnitude, snapshot X-IFU observations may reveal the presence of outflows, imprinting absorption lines around the peak of the effective area of the X-IFU. Such observations may also provide the X-ray redshift of the source and would probe the occurrence rate of outflows, their temporal variability, and their link with the kiloparsec-scale outflows running through the interstellar medium, right at the golden epoch of AGN-galaxy evolution at redshifts above two (Martocchia et al. 2017).

\section{Accounting for calibration uncertainties}

A high-resolution spectrometer such as the X-IFU will be challenging to calibrate. In this section, we investigate how uncertainties in the instrument effective area may affect the present results, which are provided so far with only statistical errors, not accounting for any systematic errors. The first test we performed was by allocating a $5 \%$ systematic error to the spectra for the configuration $3\left(A_{\mathrm{Fe}}=2, R_{\mathrm{f}}=2\right)$. The mean errors on the bestfit parameters are reported in Table 2 for configuration $3 \mathrm{~b}$. It is encouraging to see that the impact on the accuracy of the best-fit parameters is very small. Below, we introduce a more detailed analysis accounting for the X-IFU calibration requirements.

In terms of performance, the X-IFU is required to have knowledge of the shape of the effective area curve that is better than $3 \%(1 \sigma)$ across the $0.2-10 \mathrm{keV}$ range. In total, with the mirror assembly, the requirement is not to exceed $5 \%(1 \sigma$, on axis). In addition, for the X-IFU the normalization of the effective area should be known with an absolute error lower than $4 \%$ at $1 \mathrm{keV}$ (still at $1 \sigma$ ), with a contribution from the mirror of less than $6 \%$. In a first step, we restrict our exercise to X-IFU, ignoring the additional uncertainties arising from the mirror.

At low energies, the X-IFU quantum efficiency is determined by the transmission of the optical and thermal blocking filters and their supporting meshes (Barbera et al. 2018; Barret et al. 2018). Those filters are made of polymide, aluminum, and aluminum oxide. On the other hand, at high energies the quantum efficiency derives from the thickness of the absorber materials for the transition-edge sensors, currently $1.7 \mu \mathrm{m}$ of gold and $4.2 \mu \mathrm{m}$ of Bismuth (Peille et al. 2018). Here we follow the Monte Carlo simulation approach introduced by Drake et al. (2006) and followed recently by Cucchetti et al. (2018). We first generate a large number of auxiliary response files (1000) whose shape remains within the envelope of the $\pm 3 \%$ maximum-allowed shape deviation. We do this by bounding the thicknesses of the filters and the absorbers. For example, the thickness of the gold absorber is drawn from a conservative unbounded normal distribution of $\sigma=0.13 \mu \mathrm{m}$ centered around $1.7 \mu \mathrm{m}$ (Drake et al. 2006 assumed the distribution to be truncated at $\pm 1 \sigma$ ). The energies at which the maximum deviation is computed are 0.5 and $10 \mathrm{keV}$ instead of 0.2 and $12 \mathrm{keV}$. We ignore uncertainties around the edge of the response. Once the overall shape of the effective area curve is determined, its normalization is drawn from an unbounded normal distribution of mean 1 , and $\sigma=0.04$ (the same normalization applies not only at $1 \mathrm{keV}$ but throughout the whole energy band; see Fig. 10).

There are two possible approaches to estimating the errors linked to the uncertainties in the instrument response. Drake et al. (2006) proposed to start from a single simulated spectrum generated from the nominal response file, and fit it with the newly generated response files. With this approach, the statistics being the same, it better highlights the perturbations induced by the calibration uncertainties. On the one hand, the statistics of the one single spectrum to fit may have an important role in the results, as we deal with spectra with millions of counts (i.e., the fit may converge to the same best fit if the changes in the response shape are not significant enough). On the other, Cucchetti et al. (2018) faked one spectrum per newly generated response file and fit each of them with a single, nominal response file. In both cases, we wish to compare the distribution of the best-fit parameters with the distribution expected from pure Poisson statistics. We estimate the latter by faking the same number of spectra with the nominal response file, fitting them and recording the best fit parameters (i.e., the usual way of estimating best-fit errors from Monte Carlo simulations). 

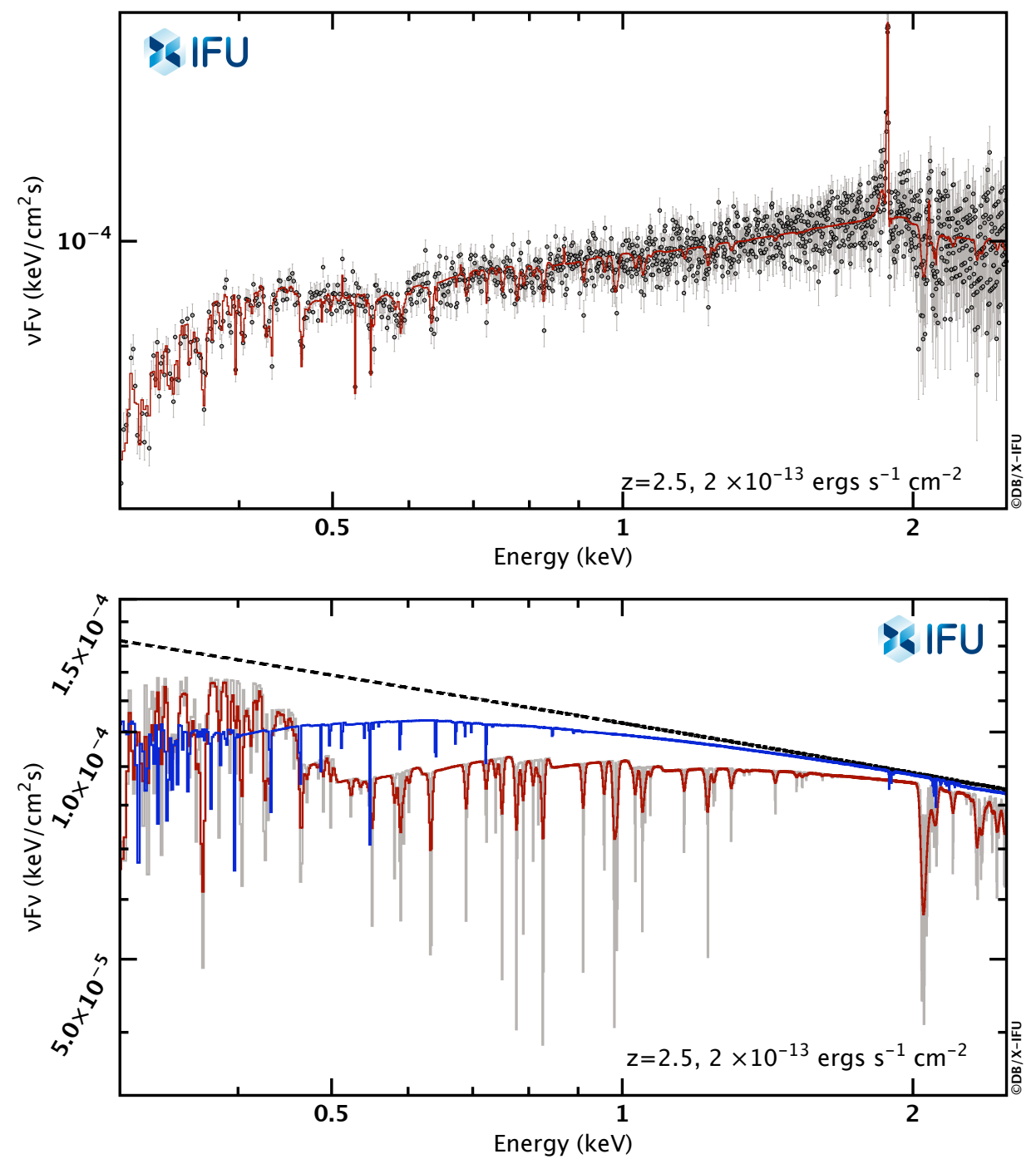

Fig. 8. Top: energy spectrum of a high-redshift AGN $(z=2.5)$ observed with X-IFU for $100 \mathrm{ks}$. Bottom: incident power-law spectrum (reflection component removed, black dashed line) to highlight the imprints of the absorbers. The low-density, low-ionization absorber is shown with the blue curve $\left(\mathrm{N}_{\mathrm{H}}=0.9 \times 10^{22} \mathrm{~cm}^{-2}, \log \xi=1.8\right)$ at a redshift of 2.46 . The high-density, high-velocity absorber $\left(\mathrm{N}_{\mathrm{H}}=7.1 \times 10^{22} \mathrm{~cm}^{-2}, \log \xi=2.9\right)$ whose redshift is 2.33 is shown without smearing in gray. A velocity broadening normalized to $1000 \mathrm{~km} \mathrm{~s}^{-1}$ at $6 \mathrm{keV}$ (rest frame) has been assumed and smears out the absorber features (red line). The black hole spin has been assumed to be 0.5 . The iron abundance has been set to 1 . Fitting such a spectrum would enable the redshift to the source and the velocity of the outflow to be measured with an extremely high accuracy of $\sim 0.001$ and $\sim 0.01$, respectively (statistical error only). This is due to the strong narrow iron line produced by the distant reflector and the large number of absorption lines due the two absorbers.

As in real life, the end user of the X-IFU will likely be provided with one single response file to fit the data that is affected by calibration uncertainties. Here we prefer the approach of Cucchetti et al. (2018). For the sake of this exercise, we simulate spectra for the so-called configuration $3(1 \mathrm{mCrab}, 100 \mathrm{ks}$, $A_{\mathrm{Fe}}=2, R_{\mathrm{f}}=2$ ), and an intermediate spin value of 0.5 . It is important to note that before all fits, the simulated spectra are optimally binned, accounting for the response file used (Kaastra \& Bleeker 2016). All fits are performed between 0.3 and $11.5 \mathrm{keV}$, and as we are interested in assessing only the distribution of best fit parameters, they all start with the model input values.

The distribution of best-fit parameters for the main reflection parameters are compared in Fig. 11 (the spin, the height of the irradiating source, the reflection fraction, and the power-law index). If systematic calibration errors were found to be important, the two distributions would differ significantly, with the distribution from Poisson statistics being narrower than the one accounting for both Poisson statistics and calibration uncertainties. As can be seen from Fig. 11, for that particular case, the calibration errors considered here (at the X-IFU level only) are small, in particular for the spin which is of prime interest here (the mean error on the spin increases from $\sim 0.02$ to $\sim 0.03$ ). It is also interesting to note that the calibration uncertainties, as modeled here, are not introducing any biases in the distributions.

Considering all calibration errors at the mirror assembly plus instrument level to arise from X-IFU alone (i.e., changing 3\% to $5 \%$ and $4 \%$ to $10 \%$ ), we repeated the simulations above, regenerating another set of 1000 response files. The systematic errors increase as expected but remain small for the spin parameter (increasing from $\sim 0.02$ to $\sim 0.04$ ). The error on the height rises at the same time from $\sim 0.2$ to $\sim 0.4 R_{\mathrm{g}}$.

It should be anticipated that the calibration errors may affect certain parameters differently to others, depending on whether they are sensitive to the low-energy part of the response or to the high-energy part, or if they relate to a continuum component or relate to a component with discrete features, such as absorption and emission lines. A more detailed analysis of the calibration requirements for X-IFU and their impact on Athena driving 


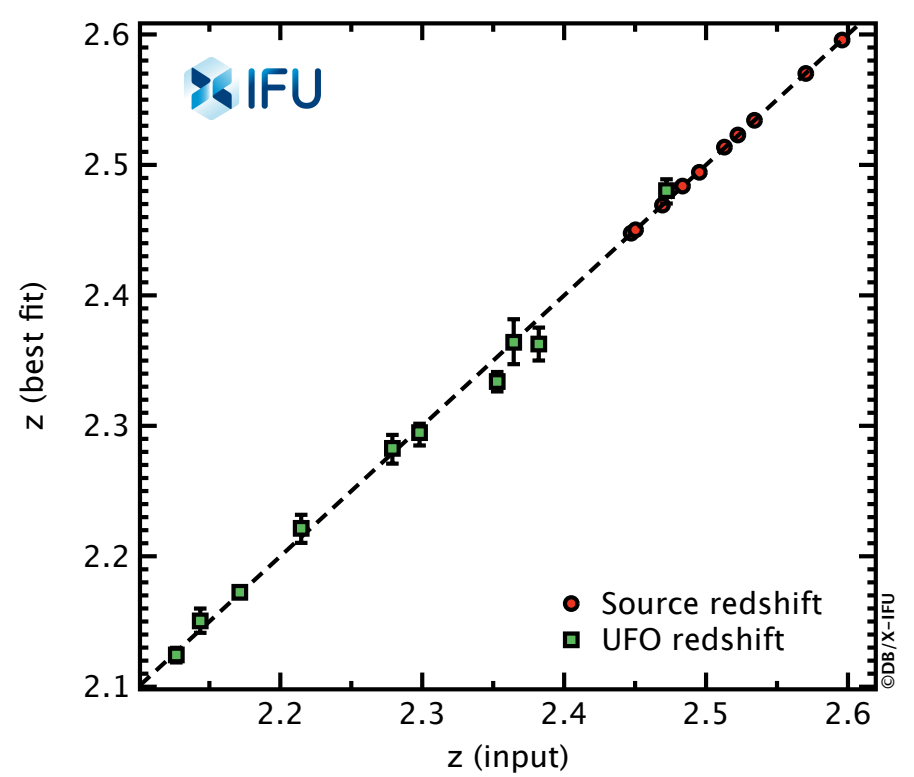

Fig. 9. Redshift of the source (filled red circles) and that of the highvelocity blueshifted absorber (filled green symbols), against their input values in the simulations. Ten source redshifts are drawn from a uniform distribution between 2.4 and 2.6, while ten blueshifts are added from a uniform distribution bounded between -0.3 and -0.05 . A large velocity broadening normalized to $3000 \mathrm{~km} \mathrm{~s}^{-1}$ has been assumed for the highvelocity absorber. The spin of the source is assumed to be 0.5 . For the reflection parameters, we assumed $R_{\mathrm{f}}=1, A_{\mathrm{Fe}}=1$. The flux of the source is $2 \times 10^{-13} \mathrm{ergs} \mathrm{cm}^{-2} \mathrm{~s}^{-1}$, and the integration of the spectrum is $100 \mathrm{ks}$. See Sect. 4 for details. The $90 \%$ confidence level errors are plotted. The errors are less than 0.001 and 0.01 for the source redshift and UFO velocity, respectively.

science cases (such as the detection of the missing baryons in the warm hot intergalactic medium) are deferred to a follow-up paper, in which we will also compare our method of assessing the systematic errors to that of Drake et al. (2006).

\section{Discussion}

We have demonstrated for the first time in a quantitative way the power of high-resolution spectroscopy in deciphering complex multi-component AGN X-ray spectra. Now, we briefly discuss the advances permitted by X-IFU in probing black hole spins, accretion-ejection physics, and the issue of iron overabundances found in AGN X-ray spectra, and conclude by a comparison with similar feasibility studies using different instrumental settings.

\subsection{New insight into the black hole spin distribution}

Measuring the distribution of spins of a large $(\gtrsim 50)$ sample of super massive black holes may tell us about their growth channels, including the relative contributions of mergers versus prolonged accretion (Berti \& Volonteri 2008; Dovciak et al. 2013). Models predict that mergers would lead to a flat spin distribution while prolonged disk-mode accretion would end up with black holes spinning rapidly. Of the few tens of AGNs with known spin values, the distribution is peaked towards high spins (Vasudevan et al. 2016; Reynolds 2019). It has been argued that this is a consequence of the fact that in a flux-limited sample, black holes with higher spins accreting at the same rate are likely to be over-represented because of their higher radiation efficiency ( $\eta=0.057$ for a nonspinning black hole to 0.32 for maximal spin $a=0.998)$. Another bias that may be present in the current data comes from the fact that higher black hole spins lead to larger $R_{\mathrm{f}}$ (Dauser et al. 2014).

In the near future, the eROSITA All-Sky Survey, reaching a 2-10 keV sensitivity limit about two orders of magnitude lower than the previous HEAO-1 All-Sky Survey (Piccinotti et al. 1982) will increase the number of objets at $z$ larger than 1 and brighter than $0.1 \mathrm{mCrab}$ from the handful known today to several hundreds (Comparat et al. 2019). Interestingly enough, recent very deep (4Ms) Chandra exposures on the Chandra Deep Field South indicate that high $z$ and nearby objects may share similar spectral properties, in particular by the presence of a broad iron line (Baronchelli et al. 2018). Therefore, with the accuracy reached on the spin measurement at the $0.1 \mathrm{mCrab}$ flux level ( 0.17 in the configuration 4 above), and thanks to its unbiased sensitivity when measuring low spins (even for relatively high source heights of $10 R_{\mathrm{g}}$ ), the results presented in this paper demonstrate that the X-IFU carries the potential to provide unprecedented constraints on the intrinsic black hole spin distribution up to $z \sim 1-2$. Such a result would have wideranging implications, such as for constraining the black hole growth models (Berti \& Volonteri 2008), but also for correcting luminosity functions or constraining black hole population synthesis models as discussed by Vasudevan et al. (2016).

\subsection{New studies of accretion and ejection flows}

The X-IFU will open a spectroscopic window to address strong gravity accretion physics and probe outflows over a range of physical parameters for the corona and reflection components of accretion disks and of AGN-driven winds, down to unprecedentedly short timescales and faint source fluxes. This will enable a new, currently unpredictable way of studying accretion and ejection phenomena. We qualitatively address a couple of such cases, considering that more extensive simulations would be required.

The X-IFU will be able to probe the height of the X-ray compact source with respect to the accretion disk down to less than a fraction of $R_{\mathrm{g}}$ and on timescales comparable to those of $\mathrm{X}$-ray source variability (see Fig. 6). In a way comparable to what is currently done in coronal mass ejections from the sun (Gou et al. 2019), it is possible that the X-ray coronae in AGNs are also formed by magnetic reconnection events on top of an accretion disk. This will lead to strong flaring, massive coronal loops, and particle acceleration. The spectral information provided by X-IFU, combined with reverberation lags between the direct and reflected emissions will probe the geometry and corona-disk structure down to the innermost regions of the accretion disk, where most of the energy is released (Dovčiak et al. 2004; Wilkins et al. 2016; Zoghbi et al. 2019).

Measuring the different parameters of AGNs and QSO-driven winds, such as their ionization parameter, column density, and velocity, with great precision is key to understanding whether such winds have sufficiently high mechanical power (typically $0.5 \%$ of the bolometric luminosity) to provide a significant contribution to AGN feedback (Hopkins \& Elvis 2010; Fabian 2012; Cappi et al. 2013). Kinetic powers being proportional to the $\mathrm{v}^{3}$, precise measurements such as the ones shown in Fig. 7, that is, yielding typical errors of less than a few percent, are mandatory. However, beside this classic argument, another new opportunity introduced in Cappi et al. (2013) is the possibility offered by X-IFU to measure not only line shifts (i.e., velocity) but also line profiles with unprecedented precision, again down to short timescales and on faint sources, i.e., in nearby Seyferts and more distant QSOs. Such information would be key to constraining the launching sites and mechanisms of the winds (see Dorodnitsyn 2009 for detailed 


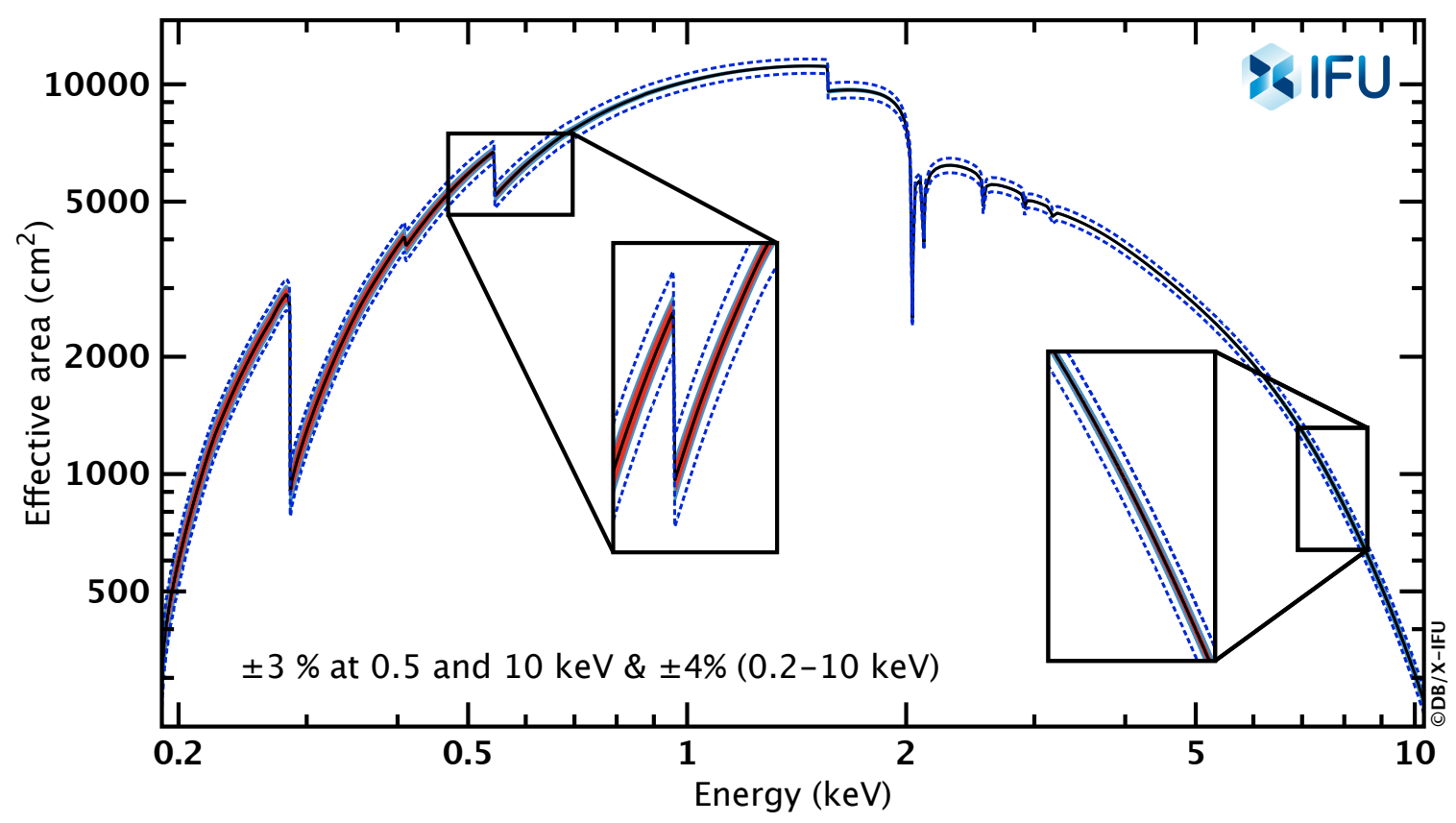

Fig. 10. Envelope of the X-IFU responses considered to assess the potential systematics related to calibration uncertainties. The $3 \%(1 \sigma)$ on the relative broad band effective area is shown in filled red around the nominal response which is indicated with a solid black line. The $4 \%(1 \sigma)$ on the absolute effective area knowledge is delimited by the blue dashed line and comes on top of the previous one. 1000 response files are drawn from within the envelope, assuming non truncated normal distributions. See Sect. 5 for details about the method.

simulations of such profiles, and Chartas et al. 2016 for a tentative application to Chandra data).

Done et al. (2007) and Nardini et al. (2015) have shown that the broad FeK emission lines combined with the strong absorption features at higher energies seen in some bright nearby AGNs may well be interpreted as P-Cygni line profiles produced by a spherically symmetric wind or shell. Similar P-Cygni profiles should be seen for all absorption lines but with different shapes and different time variations for the different absorbers. In addition, a realistic flow will be rather radially extended with a distribution of kinematical, ionization, and dynamical properties along the line of sight, leading to even more complex absorption profiles (Proga \& Kurosawa 2010; Giustini \& Proga 2012). Simulating such complex spectra with X-IFU goes beyond the scope of this paper, but clearly X-IFU holds the potential to provide key insights into the wind properties.

\subsection{Iron overabundance, and disk inclination and ionization}

Inferred iron overabundances from X-ray reflection spectroscopy is one of the most intriguing results, casting doubt on the reported spin values given the tight relation between reflection parameters and iron abundances. This has motivated the revision of reflection models towards densities above the currently used values: those densities being expected in the vicinity of black holes (García et al. 2016, 2019a). Application of high-density models to a few selected objects has already shown that the iron abundance recovered was significantly lower than the one obtained with lower-density disk reflection models (Tomsick et al. 2018; Jiang et al. 2019). Those models that are currently under development have clear signatures at energies below $1 \mathrm{keV}$. In particular, the enhancement of free-free heating in the atmosphere of the disk, increasing with increasing density, leads to a soft excess. These high-density models will be easily testable with X-IFU, which will measure the iron abundance down to solar, together with the reflection component with high accuracy (see Tables 2 and 3).
We have also shown (Sect. 3.7) that the disk inclination and ionization will be well constrained. This is very important because inclination measurements could allow comparison of inner disk inclinations to those for the host galaxy stellar disk, thereby putting constraints on the way AGNs are fueled. Material propagating inward through the galactic disk or via minor mergers is expected to leave imprints on the average respective alignment of the objects concerned (Middleton et al. 2016).

Understanding the degree to which the reflection component is ionized is also an open and debated issue. The FeK line profile in principle carries sensitive information on the disk ionization state, but in practice it is often degenerate with the other free parameters of the line profile. As a result, the soft energy band is key to constraining the amount of ionization for the reflection component as shown in the lower left panel of Fig. 1 where the disk soft emission becomes quickly very significant at intermediate up to high ionization levels. As a note of caution, it is worth stating that in our lamp-post model, the ionisation is assumed to be radially constant. Ideally it should be calculated self-consistently with the radial density to account for the centrally peaked illumination expected in a relativistic accretion disk model (see Martocchia et al. 2002; Svoboda et al. 2012 and in particular Kammoun et al. 2019 for a consideration of this effect, including also X-IFU simulations).

\subsection{Comparison with other instruments and simulations}

Feasibility studies have so far been carried out, considering $\mathrm{X}$-ray spectra with limited spectral resolution, that is, $\sim 100 \mathrm{eV}$ at $6-7 \mathrm{keV}$, as provided by XMM-Newton EPIC instruments for example (Strüder et al. 2001), combined with hard X-ray data enabling sampling of the smooth Compton reflection bump above $10 \mathrm{keV}$, as provided by NuSTAR for example (Harrison et al. 2013). We briefly discuss here how these studies compare to those presented in this paper. 

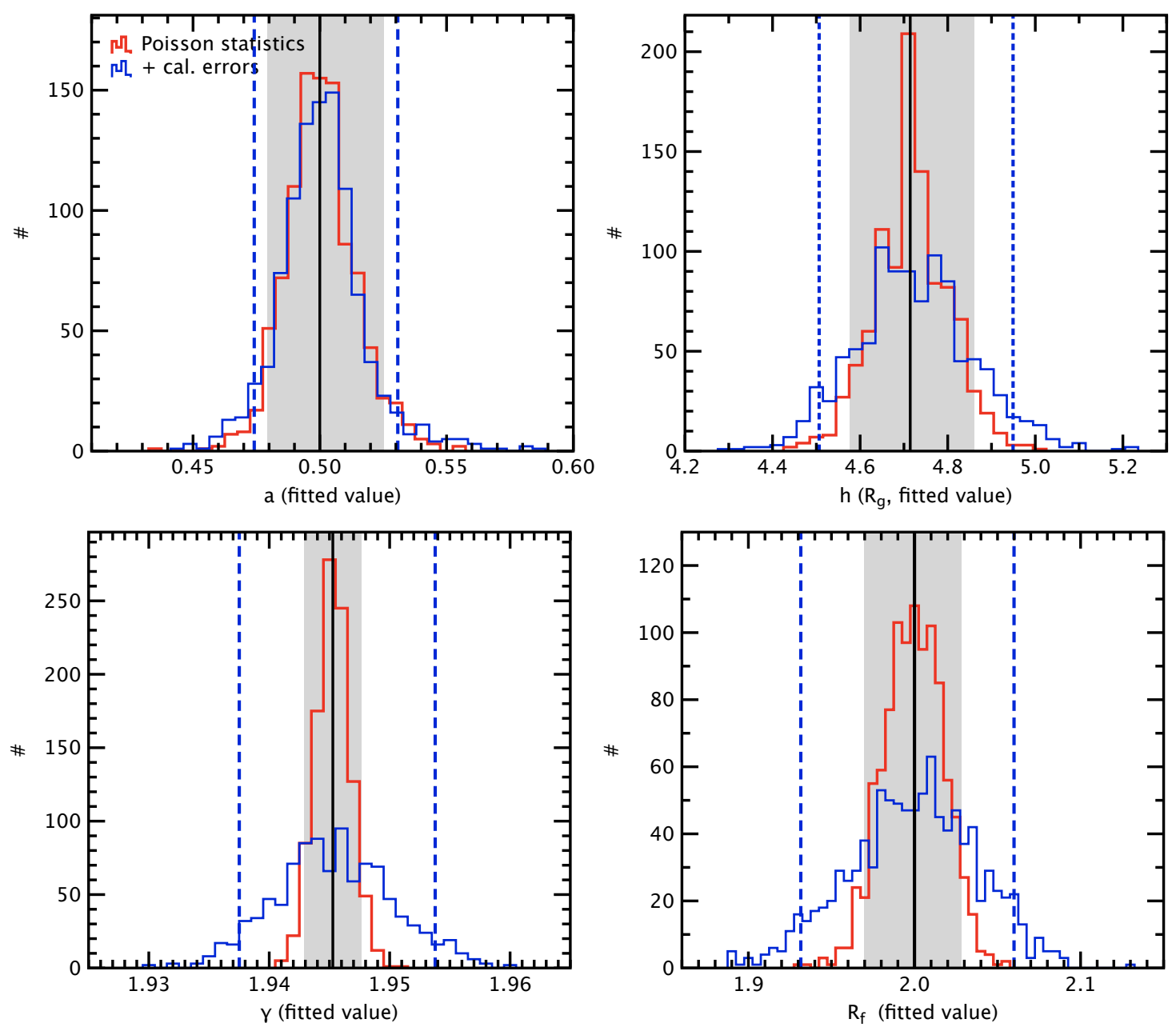

Fig. 11. Histogram distribution of best-fit parameters arising from Poisson statistics alone (in red, the $90 \%$ quantile is indicated with the gray area) and from Poisson statistics and calibration errors (in blue, the $90 \%$ quantile is delimited with the blue dashed line). Top-left: spin parameter. Top-right: height of the irradiating source. Bottom-left: power law index. Bottom-right: reflection fraction. The case simulated here corresponds to a spin of 0.5 , a $1 \mathrm{mCrab}$ source observed for $100 \mathrm{ks}$, with a reflection fraction set to two. As can be seen, at the level of calibration errors considered here, the spin parameter and the height of the X-ray source do not suffer from any significant systematic error, while the systematic errors on the power-law index and reflection fraction are larger, but remain small. No biases are introduced in all cases; see Sect. 5.

Kammoun et al. (2018) conducted a similar analysis to ours, simulating XMM-Newton EPIC-PN and NuSTAR spectra in the range of $1-3 \mathrm{mCrab}$ fluxes. Beside relativistic reflection, they considered a warm absorber and two layers of partially covering neutral absorbers, cold reflection, and thermal emission from the galaxy, thus introducing complexity in their spectral model similar to ours. The success rate of measuring their spin is about $50 \%$ (over 60 fits). This rate increases to $100 \%$ for spins larger than 0.8 and a lamp-post height lower than five gravitational radii (because this configuration imprints stronger, easier-to-detect relativistic distortions to the spectrum; see also Choudhury et al. 2017). On the other hand, the success rate drops to zero if the height of the irradiating source is at a distance larger than $5 R_{\mathrm{g}}$. As demonstrated above, $\mathrm{X}$-IFU can measure spins all across the range investigated, and this even for small reflection fractions and iron abundance of 1 , and source height up to $10 R_{\mathrm{g}}$. Interestingly, Kammoun et al. (2018), considering two of their failed simulations, with the height of irradiating source at 11 and $18 R_{\mathrm{g}}$, noticed that Athena WFI simulations would not be more successful (despite the much improved statistics), concluding that this was likely due to the relatively poor sampling of the reflection hump above $10 \mathrm{keV}$. Because the Compton hump is not properly sampled by X-IFU either, it may be more likely due to an overly low reflection fraction (due to the large source heights considered). We have repeated the simulations of Configuration $1\left(A_{\mathrm{Fe}}=1\right.$ and $\left.R_{\mathrm{f}}=1\right)$ using the WFI response files ${ }^{4}$ and found that the accuracy by which WFI recovers the reflection parameters is a factor of approximately two less than X-IFU, despite the higher effective area of the WFI at high energy ( $\sim 25 \%$ around $6 \mathrm{keV}$ ). Taking advantage of its better spectral resolution, at the same time, the X-IFU recovers the parameters of the absorbers with error bars that are between a factor of three to four smaller (see configuration $1 \mathrm{~b}$ in Table 2).

Bonson \& Gallo (2016) considered a model based on relxill only (i.e., without absorbers and cold reflection; see Choudhury et al. 2017 for a discussion on their fitting scheme). They simulated spectra with XMM-Newton EPIC-PN and NuSTAR for the brightest seyferts, and found that the spin parameter could only be accurately measured for the most rapidly rotating super-massive black holes (i.e., $a>0.8$ to about \pm 0.10 ). The error on the spin would reach $\sim 0.30$ at $a=0$ for $R_{\mathrm{f}}=5$, a value

4 The response files were downloaded from http://www.mpe.mpg. de/ATHENA-WFI/response_matrices.html and the date of the version used is November 2017. See Meidinger et al. (2018) for a recent description of the WFI instrument. 
not considered here. Interestingly enough, in their simulations they found that the addition of NuSTAR hard X-ray data did not improve the spin determination (see Fig. 7 of Bonson \& Gallo 2016). At first sight, the simulations performed here do not seem heavily impacted by the lack of hard X-ray data (above $10 \mathrm{keV}$ ), possibly because there is sufficient information across the X-IFU band pass, in particular in the soft X-ray band where the ionized reflection component contributes significantly.

Following up on this, it is worth noting that the powerlaw index is extremely well constrained within our simulations, despite the model complexity. The assumption of a straight power law in the X-IFU band pass is correct for any plausible high-energy cutoffs (above tens of keV). García et al. (2015) showed that the high-energy cutoff up to even $1 \mathrm{MeV}$ can be constrained using X-ray data below $100 \mathrm{keV}$ by modeling the reflection component alone. This is due to the fact that the reflection spectrum, imprinted by fluorescent lines and other atomic features, depends sensitively on the shape of the emission spectrum of the irradiating source. We have repeated the simulations in configuration $3\left(1 \mathrm{mCrab}, 100 \mathrm{ks}, R_{\mathrm{f}}=2, A_{\mathrm{Fe}}=2\right)$, leaving the energy cutoff as a free parameter, allowing it to vary between 50 and $200 \mathrm{keV}$ (drawing the initial 50 values from a uniform distribution). Such a cutoff range is consistent with the latest $S$ wift/XRTNuSTAR observations of type 1 AGN (Molina et al. 2019, see also Ricci et al. 2017 and references therein). The mean 90\% confidence level error on the energy cutoff derived from the X-IFU simulations is $\sim 15 \mathrm{keV}$ over the $50-200 \mathrm{keV}$ range, with a tendency for the errors to increase at the upper end of the range. This indeed suggests that meaningful constraints can be obtained on the highenergy cutoff from the X-ray data alone. This also means that combining X-IFU data with comparably sensitive hard X-ray data, for example from the High-Energy X-ray Probe (HEX-P) proposed as a complementary mission to Athena (Madsen et al. 2018), would set very tight constraints on the reflection parameters by precisely measuring both the energy cutoff and the Compton hump. It is also worth noting that the shape of the Compton hump, being independent of parameters such as the iron abundance or the disk ionization would help in removing model degeneracies, in case data are more complex than those simulated here (as they likely will be).

Finally, Choudhury et al. (2017) tested the relxill model with simulated NuSTAR data, and assumptions more extreme than ours; for example $R_{\mathrm{f}}$ values up to 10 and iron overabundance also up to 10 . They also considered NuSTAR spectra accumulated over $100 \mathrm{ks}$ and delivering between 1 and 10 millions counts. For the model considered here and a source of $1 \mathrm{mCrab}$, the rate expected in the one NuSTAR module is $\sim 0.5$ counts $\mathrm{s}^{-1}$ between 3 and $70 \mathrm{keV}^{5}$, meaning that the fluxes they considered would correspond to 20 to $200 \mathrm{mCrab}$ for X-IFU: a flux regime not explored in this paper (and in which there are just a couple of AGNs). They found that better constraints are obtained for smaller height of the irradiating source and larger reflection fractions, yet the errors that they obtained in the most favorable conditions exceed that obtained here in our more realistic and complex setting by at least one order of magnitude. To take an example, for a spin parameter input of zero, and $R_{\mathrm{f}}=1$ and $h=3 R_{\mathrm{g}}$, the $90 \%$ dispersion among the simulations goes from $\sim-0.5$ to $\sim 0.25$, while for sources 200 times fainter, the X-IFU would reach an error of $\leq 0.1$.

\subsection{Comparison with XRISM-Resolve}

The X-ray Imaging and Spectroscopy Mission (XRISM), a JAXA/NASA collaborative mission, with ESA participation, is

\footnotetext{
5 Response files were downloaded from https://www.nustar. caltech.edu/page/response_files
}

expected to launch around 2021 (Tashiro et al. 2018). It will carry Resolve, a soft X-ray spectrometer, which combines a lightweight soft X-ray telescope with an X-ray calorimeter spectrometer to provide nondispersive $5-7 \mathrm{eV}$ energy resolution in the $0.3-12 \mathrm{keV}$ bandpass. Opening the way to broadband highresolution X-ray spectroscopy, which we have seen to be critical for the science objectives of this paper, it will be interesting to compare how Resolve will perform compared to X-IFU, despite its lower effective area (about a factor of $\sim 45$ at $1 \mathrm{keV}$ and a factor of $\sim 5$ at $6 \mathrm{keV}$ ), and this, at least for the brightest objects. For the sake of this simple comparison and focussing on the spin measurements, we simulated a $5 \mathrm{mCrab}$ source in the so-called conservative configuration 1 above $\left(R_{\mathrm{f}}=1, A_{\mathrm{Fe}}=1\right)$. We generated 50 spectra with a constant spin spacing between 0 and 0.995 and with an integration time of $100 \mathrm{ks}$. Setting a favorable case, we ignored the background and initiated the fit to the model input parameters. The error on the spin parameter was then computed. In about $\sim 15 \%$ of the simulations, the fitted spin parameter pegged at the hard limit. The mean error on the spin is $\sim 0.3$. With the same settings, the mean error on the spin from X-IFU observations would be $\sim 0.04$.

To summarize, the comparison with the three feasibility studies similar to the one presented here, as well as the comparison with the XRISM-Resolve above, clearly demonstrate the advances the X-IFU will permit over existing and future instrumentations.

\section{Conclusions}

The Athena X-IFU, as currently designed, is predicted to be transformational in many fields of astrophysics, and so will Athena overall, by the complementarity of its science payload (Nandra et al. 2013; Barret et al. 2013; Barcons et al. 2017; Guainazzi \& Tashiro 2018). Here we have demonstrated the rather unique and outstanding capabilities of X-IFU in probing AGN spins, AGN surroundings, accretion disk physics, winds, and outflows from local to more distant AGNs using a stateof-the-art reflection model in a lamp-post geometrical configuration. The leap in sensitivity provided by X-IFU derives from its excellent spectral resolution, high throughput, and broadband coverage. More feasibility studies of this type, possibly combining spectral-timing analysis, extending the range of models to be tested, the range of reflection geometries, and the range of objects to be considered (e.g., X-ray binaries), should be performed to further assess and quantify its unique capabilities. The methodology presented here may also serve this purpose.

Acknowledgements. The authors wish to thank the anonymous referee for useful comments. DB acknowledges useful discussions with Thomas Dauser, Edoardo Cucchetti, Chris Done, Jeremy Drake and Etienne Pointecouteau. DB and MC thank Laura Brenneman, Francisco Carrera, Mauro Dadina, Javier Garcia, Matteo Guainazzi, Jelle Kaastra, Elias Kammoun, Giovanni Miniutti, Jon Miller, and Jiri Svoboda for their useful suggestions and comments on an earlier version of the paper. Special thanks to Edoardo Cucchetti and the computer support team at IRAP (Elodie Bourrec and Cédric Hillembrand) for providing DB with the cluster resources required to carry out these time consuming simulations. MC acknowledges financial support from the Italian Space Agency under agreement ASI-INAF n.2017-14-H.O.and 2018-11-HH.0. DB acknowledges support from the French Space Agency (CNES). DB wishes to dedicate this paper to his beloved mother who passed away during the preparation of this work.

\section{References}

Arnaud, K. A. 1996, Astronomical Data Analysis Software and Systems V, 101, 17

Barbera, M., Lo Cicero, U., Sciortino, L., et al. 2018, Space Telescopes and Instrumentation 2018: Ultraviolet to Gamma Ray, 10699, 106991R 
Barcons, X., Barret, D., Decourchelle, A., et al. 2017, Astron. Nachr., 338, 153 Baronchelli, L., Nandra, K., \& Buchner, J. 2018, MNRAS, 480, 2377

Barret, D., Nandra, K., Barcons, X., et al. 2013, SF2A-2013: Proceedings of the Annual meeting of the French Society of Astronomy and Astrophysics, 447

Barret, D., Lam Trong, T., den Herder, J. W., et al. 2018, Space Telescopes and Instrumentation 2018: Ultraviolet to Gamma Ray, 10699, $106991 \mathrm{G}$

Berti, E., \& Volonteri, M. 2008, ApJ, 684, 822

Blustin, A. J., Page, M. J., Fuerst, S. V., Branduardi-Raymont, G., \& Ashton, C. E. 2005, A\&A, 431, 111

Boller, T., Fabian, A. C., Sunyaev, R., et al. 2002, MNRAS, 329, L1

Bonson, K., \& Gallo, L. C. 2016, MNRAS, 458, 1927

Brenneman, L. 2013, Measuring the Angular Momentum of Supermassive Black Holes (SpringerBriefs in Astronomy)

Brenneman, L. W., \& Reynolds, C. S. 2006, ApJ, 652, 1028

Cappi, M., Done, C., Behar, E., et al. 2013, ArXiv e-prints [arXiv:1306.2330]

Cappi, M., De Marco, B., Ponti, G., et al. 2016, A\&A, 592, A27

Cash, W. 1979, ApJ, 228, 939

Chartas, G., Cappi, M., Hamann, F., et al. 2016, ApJ, 824, 53

Choudhury, K., García, J. A., Steiner, J. F., \& Bambi, C. 2017, ApJ, 851, 57

Comparat, J., Merloni, A., Salvato, M., et al. 2019, MNRAS, 1335

Crummy, J., Fabian, A. C., Gallo, L., \& Ross, R. R. 2006, MNRAS, 365, 1067

Cucchetti, E., Pajot, F., Pointecouteau, E., et al. 2018, Space Telescopes and Instrumentation 2018: Ultraviolet to Gamma Ray, 10699, 1069940

Dadina, M., Vignali, C., Cappi, M., et al. 2018, A\&A, 610, L13

Dauser, T., Garcia, J., Wilms, J., et al. 2013, MNRAS, 430, 1694

Dauser, T., García, J., Parker, M. L., Fabian, A. C., \& Wilms, J. 2014, MNRAS, 444, L100

Dauser, T., García, J., Walton, D. J., et al. 2016, A\&A, 590, A76

de La Calle Pérez, I., Longinotti, A. L., Guainazzi, M., et al. 2010, A\&A, 524, A50

De Marco, B., Ponti, G., Cappi, M., et al. 2013, MNRAS, 431, 2441

Done, C. 2010, ArXiv e-prints [arXiv:1008.2287]

Done, C., Sobolewska, M. A., Gierliński, M., \& Schurch, N. J. 2007, MNRAS 374, L15

Dorodnitsyn, A. V. 2009, MNRAS, 393, 1433

Dovčiak, M., Karas, V., \& Yaqoob, T. 2004, ApJS, 153, 205

Dovciak, M., Matt, G., Bianchi, S., et al. 2013, ArXiv e-prints [arXiv:1306.2331]

Drake, J. J., Ratzlaff, P., Kashyap, V., et al. 2006, Proc. SPIE, 6270, 62701I

Emmanoulopoulos, D., Papadakis, I. E., Dovčiak, M., \& McHardy, I. M. 2014 MNRAS, 439, 3931

Evans, I. N., Allen, C., Anderson, C. S., et al. 2019, AAS/High Energy Astrophysics Division, 17, 114.01

Fabian, A. C. 2012, ARA\&A, 50, 455

Fabian, A. C. 2016, Astron. Nachr., 337, 375

Fabian, A. C., Zoghbi, A., Ross, R. R., et al. 2009, Nature, 459, 540

Fabian, A. C., Alston, W. N., Cackett, E. M., et al. 2017, Astron. Nachr., 338 269

Gallo, L. C., \& Fabian, A. C. 2011, MNRAS, 418, L59

Gallo, L. C., \& Fabian, A. C. 2013, MNRAS, 434, L66

Gallo, L. C., Wilkins, D. R., Bonson, K., et al. 2015, MNRAS, 446, 633

Gallo, L. C., Gonzalez, A. G., Waddell, S. G. H., et al. 2019, MNRAS, 484, 4287

García, J., \& Kallman, T. R. 2010, ApJ, 718, 695

García, J., Dauser, T., Reynolds, C. S., et al. 2013, ApJ, 768, 146

García, J., Dauser, T., Lohfink, A., et al. 2014, ApJ, 782, 76

García, J. A., Dauser, T., Steiner, J. F., et al. 2015, ApJ, 808, L37

García, J. A., Fabian, A. C., Kallman, T. R., et al. 2016, MNRAS, 462, 751

García, J. A., Kallman, T. R., Bautista, M., et al. 2018, Workshop on Astrophysical Opacities, 515, 282

García, J. A., Bachetti, M., Ballantyne, D. R., et al. 2019a, BAAS, 51, 284

García, J. A., Kara, E., Walton, D., et al. 2019b, ApJ, 871, 88

Georgakakis, A., Carrera, F., Lanzuisi, G., et al. 2013, ArXiv e-prints [arXiv:1306.2328]

Gierliński, M., \& Done, C. 2006, MNRAS, 371, L16

Giustini, M., \& Proga, D. 2012, ApJ, 758, 70

Gofford, J., Reeves, J. N., Tombesi, F., et al. 2013, MNRAS, 430, 60

Gou, T., Liu, R., Kliem, B., Wang, Y., \& Veronig, A. M. 2019, Sci. Adv., 5, eaau 7004

Guainazzi, M., \& Tashiro, M. S. 2018, ArXiv e-prints [arXiv:1807.06903]

Guainazzi, M., Bianchi, S., \& Dovčiak, M. 2006, Astron. Nachr., 327, 1032

Harrison, F. A., Craig, W. W., Christensen, F. E., et al. 2013, ApJ, 770, 103

Hopkins, P. F., \& Elvis, M. 2010, MNRAS, 401, 7

Humphrey, P. J., Liu, W., \& Buote, D. A. 2009, ApJ, 693, 822

Hurkett, C. P., Vaughan, S., Osborne, J. P., et al. 2008, ApJ, 679, 587

Jiang, J., Fabian, A. C., Wang, J., et al. 2019, MNRAS, 484, 1972

Kaastra, J. S. 2017a, Astron. Nachr., 338, 146

Kaastra, J. S. 2017b, A\&A, 605, A51
Kaastra, J. S., \& Bleeker, J. A. M. 2016, A\&A, 587, A151

Kaastra, J. S., Kriss, G. A., Cappi, M., et al. 2014, Science, 345, 64

Kallman, T., \& Bautista, M. 2001, ApJS, 133, 221

Kammoun, E. S., Nardini, E., \& Risaliti, G. 2018, A\&A, 614, A44

Kammoun, E. S., Domček, V., Svoboda, J., Dovčiak, M., \& Matt, G. 2019, MNRAS, 485, 239

Kara, E., Alston, W., \& Fabian, A. 2016, Astron. Nachr., 337, 473

Laha, S., Guainazzi, M., Dewangan, G. C., Chakravorty, S., \& Kembhavi, A. K. 2014, MNRAS, 441, 2613

Laha, S., Smith, R., Tzanavaris, P., et al. 2019, BAAS, 51, 75

Madsen, K. K., Harrison, F., Broadway, D., et al. 2018, Space Telescopes and Instrumentation 2018: Ultraviolet to Gamma Ray, 10699, 106996M

Madsen, K. K., Natalucci, L., Belanger, G., et al. 2019, ArXiv e-prints [arXiv:1901.00934]

Magdziarz, P., Blaes, O. M., Zdziarski, A. A., Johnson, W. N., \& Smith, D. A. 1998, MNRAS, 301, 179

Martocchia, A., Matt, G., \& Karas, V. 2002, A\&A, 383, L23

Martocchia, S., Piconcelli, E., Zappacosta, L., et al. 2017, A\&A, 608, A51

Mehdipour, M., Kaastra, J. S., Kriss, G. A., et al. 2015, A\&A, 575, A22

Meidinger, N., Nandra, K., \& Plattner, M. 2018, Space Telescopes and Instrumentation 2018: Ultraviolet to Gamma Ray, 10699, 106991F

Middei, R., Bianchi, S., Petrucci, P.-O., et al. 2019, MNRAS, 483, 4695

Middleton, M. J., Parker, M. L., Reynolds, C. S., Fabian, A. C., \& Lohfink, A. M. 2016, MNRAS, 457, 1568

Miller, L., \& Turner, T. J. 2013, ApJ, 773, L5

Miller, L., Turner, T. J., \& Reeves, J. N. 2009, MNRAS, 399, L69

Miller, L., Turner, T. J., Reeves, J. N., \& Braito, V. 2010, MNRAS, 408, 1928

Miniutti, G., Ponti, G., Greene, J. E., et al. 2009, MNRAS, 394, 443

Molina, M., Malizia, A., Bassani, L., et al. 2019, MNRAS, 484, 2735

Nandra, K., George, I. M., Mushotzky, R. F., Turner, T. J., \& Yaqoob, T. 1997, ApJ, 477, 602

Nandra, K., O’Neill, P. M., George, I. M., \& Reeves, J. N. 2007, MNRAS, 382, 194

Nandra, K., Barret, D., Barcons, X., et al. 2013, ArXiv e-prints [arXiv:1306.2307]

Nardini, E., Reeves, J. N., Gofford, J., et al. 2015, Science, 347, 860

Oh, K., Koss, M., Markwardt, C. B., et al. 2018, VizieR Online Data Catalog: II/235

Parker, M. L., Matzeu, G. A., Guainazzi, M., et al. 2018a, MNRAS, 480, 2365

Parker, M. L., Miller, J. M., \& Fabian, A. C. 2018b, MNRAS, 474, 1538

Peille, P., den Hartog, R., Macculi, C., et al. 2018, Space Telescopes and Instrumentation 2018: Ultraviolet to Gamma Ray, 10699, 106994K

Petrucci, P.-O., Ursini, F., De Rosa, A., et al. 2018, A\&A, 611, A59

Piccinotti, G., Mushotzky, R. F., Boldt, E. A., et al. 1982, ApJ, 253, 485

Ponti, G., Papadakis, I., Bianchi, S., et al. 2012, A\&A, 542, A83

Pounds, K. A., Reeves, J. N., King, A. R., et al. 2003, MNRAS, 345, 705

Proga, D., \& Kurosawa, R. 2010, Accretion and Ejection in AGN: A Global View, 427, 41

Reeves, J. N., O’Brien, P. T., \& Ward, M. J. 2003, ApJ, 593, L65

Reeves, J., Done, C., Pounds, K., et al. 2008, MNRAS, 385, L108

Reynolds, C. S. 2019, Nat. Astron., 3, 41

Reynolds, C. S., Brenneman, L. W., Lohfink, A. M., et al. 2012, ApJ, 755, 88

Ricci, C., Trakhtenbrot, B., Koss, M. J., et al. 2017, ApJS, 233, 17

Risaliti, G., Harrison, F. A., Madsen, K. K., et al. 2013, Nature, 494, 449

Rosen, S. R., Webb, N. A., Watson, M. G., et al. 2019, VizieR Online Data Catalog: IX/055

Svoboda, J., Dovčiak, M., Goosmann, R. W., et al. 2012, A\&A, 545, A106

Strüder, L., Briel, U., Dennerl, K., et al. 2001, A\&A, 365, L18

Tanaka, Y., Nandra, K., Fabian, A. C., et al. 1995, Nature, 375, 659

Tanaka, Y., Boller, T., Gallo, L., Keil, R., \& Ueda, Y. 2004, PASJ, 56, L9

Tanimoto, A., Ueda, Y., Odaka, H., et al. 2019, ApJ, 877, 95

Tashiro, M., Maejima, H., Toda, K., et al. 2018, Space Telescopes and Instrumentation 2018: Ultraviolet to Gamma Ray, 1069922

Tombesi, F., Cappi, M., Reeves, J. N., et al. 2010, A\&A, 521, A57

Tombesi, F., Cappi, M., Reeves, J. N., et al. 2011, ApJ, 742, 44

Tombesi, F., Cappi, M., Carrera, F., et al. 2019, BAAS, 51, 103

Tomsick, J. A., Parker, M. L., García, J. A., et al. 2018, ApJ, 855, 3

Vasudevan, R. V., Fabian, A. C., Reynolds, C. S., et al. 2016, MNRAS, 458, 2012

Verner, D. A., Yakovlev, D. G., Band, I. M., et al. 1993, At. Data Nucl. Data Tables, 55, 233

Walton, D. J., Nardini, E., Gallo, L. C., et al. 2019, MNRAS, 484, 2544

Wilkins, D. R., Cackett, E. M., Fabian, A. C., \& Reynolds, C. S. 2016, MNRAS, 458, 200

Wilms, J., Allen, A., \& McCray, R. 2000, ApJ, 542, 914

Zoghbi, A., Fabian, A. C., Uttley, P., et al. 2010, MNRAS, 401, 2419

Zoghbi, A., Uttley, P., \& Fabian, A. C. 2011, MNRAS, 412, 59

Zoghbi, A., Wilkins, D., Brenneman, L., et al. 2019, BAAS, 51, 62 
D. Barret and M. Cappi: High resolution X-ray spectroscopy of accreting supermassive black holes

\section{Appendix A: Biases in $\chi^{2}$ fitting}

$\chi^{2}$ statistics is often used as a fitting metric, although its limitations are known, especially in the (e.g. Madsen et al. 2019) here after low-count-rate regime. As discussed by Humphrey et al. (2009), even in the high-count-rate regime (when the counts per bin become typically larger than $\sim 20), \chi^{2}$ fitting leads to biased parameter estimates, unless the number of data bins is far smaller than the square root of the number of counts in the spectrum (which is not the case for most simulations presented here). The bias may be comparable to, or even exceed, the statistical error. We have repeated the configuration 1 simulation, replacing the optimal binning scheme of Kaastra \& Bleeker (2016) with a standard grouping scheme ensuring that each spectral bin has at least 20 counts. We used $\chi^{2}$ statistics. Of all the 16 free parameters of the fit, the photon index of the power law has a very small statistical error (0.003 in Table 2). In Fig. A.1, the best-fit powerlaw index is reported against the input power-law index. As can be seen, a bias is present towards recovering steeper indexes, and the bias exceeds the statistical error. The bias is still present when the data are grouped further to a minimum of 50 counts per bin. A similar bias was present in the simulations reported by Choudhury et al. (2017). No such bias is present in our fits based on cstat, as shown in Fig. 3. To conclude, for X-IFU data, it is recommended to always use cstat in fitting spectra; see also Kaastra (2017b) on how cstat can be used for statistical tests, such as assessing the goodness of fit of a spectral model, as used here.

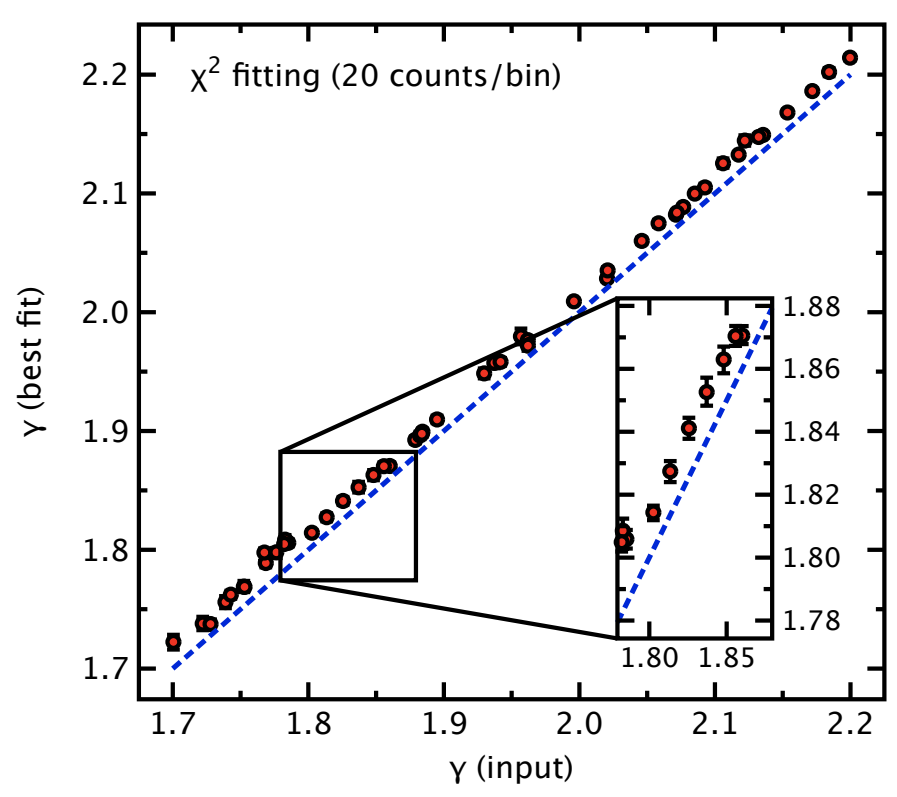

Fig. A.1. Best-fit power-law index with its $90 \%$ confidence level error plotted against the input value to show that with $\chi^{2}$ fitting a bias larger than the statistical error is introduced. The input power-law index of the irradiating source is drawn uniformly between 1.7 and 2.2 (50 values). Before fitting, the spectra are binned to have a minimum number of 20 counts per bin. No such bias is seen when the cstat is used, as indicated in Fig. 3 (top-right panel). 